\title{
Efficient precise knockin with a double cut HDR donor after CRISPR/Cas9-mediated double-stranded DNA cleavage
}

\author{
Jian-Ping Zhang ${ }^{1,2+}$, Xiao-Lan Li ${ }^{1,2+}$, Guo-Hua Li ${ }^{1,2}$, Wanqiu Chen ${ }^{7}$, Cameron Arakaki ${ }^{7}$, Gary D. Botimer ${ }^{8}$,

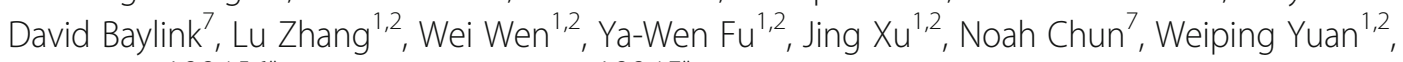 \\ Tao Cheng ${ }^{1,2,3,4,5,6^{*}}$ and Xiao-Bing Zhang ${ }^{1,2,3,4,7^{*}}$
}

\begin{abstract}
Background: Precise genome editing via homology-directed repair (HDR) after double-stranded DNA (dsDNA) cleavage facilitates functional genomic research and holds promise for gene therapy. However, HDR efficiency remains low in some cell types, including some of great research and clinical interest, such as human induced pluripotent stem cells (iPSCs).

Results: Here, we show that a double cut HDR donor, which is flanked by single guide RNA (sgRNA)-PAM sequences and is released after CRISPR/Cas9 cleavage, increases HDR efficiency by twofold to fivefold relative to circular plasmid donors at one genomic locus in 293 T cells and two distinct genomic loci in iPSCs. We find that a $600 \mathrm{bp}$ homology in both arms leads to high-level genome knockin, with $97-100 \%$ of the donor insertion events being mediated by HDR. The combined use of CCND1, a cyclin that functions in G1/S transition, and nocodazole, a G2/M phase synchronizer, doubles HDR efficiency to up to 30\% in iPSCs.
\end{abstract}

Conclusions: Taken together, these findings provide guidance for the design of HDR donor vectors and the selection of HDR-enhancing factors for applications in genome research and precision medicine.

Keywords: CRISPR, Genome editing, Knockin, Homology-directed repair (HDR), Non-homologous end joining (NHEJ), Donor design, 293 T, Human induced pluripotent stem cells

\section{Background}

The ability to precisely edit genomes endows scientists with a powerful tool to interrogate the functionalities of any pieces of DNA in the genome of any species and it may also lead to the development of new therapies that can potentially cure numerous genetic diseases [1-3]. However, precise gene editing by homologous recombination is very inefficient, unless a DNA double-stranded break (DSB) is created at the targeting site, which increases homology-directed repair (HDR) mediated gene editing efficiency by $\sim 1000$-fold [4-6]. To induce DSB at a desired site, several technologies have been developed over the past decade, including zinc-finger nucleases

\footnotetext{
*Correspondence: chengtao@ihcams.ac.cn; xzhang@llu.edu

${ }^{\dagger}$ Equal contributors

${ }^{1}$ State Key Laboratory of Experimental Hematology, Tianjin, China Full list of author information is available at the end of the article
}

(ZFN), transcription activator-like effector nucleases (TALEN), and the clustered regularly interspaced short palindromic repeats (CRISPR)/CRISPR-associated protein-9 nuclease (Cas9) system [1]. The CRISPR-Cas9 system has caught widespread attention due to its robust performance, simple vector construction, and multiplexability in manipulating genes $[7,8]$.

CRISPR is an adoptive immune system evolved in bacteria and archaea to fight against invading agents such as bacteriophages or plasmids [9]. Diverse CRISPR systems have been adapted for use in editing mammalian genomes [10-12]. Currently the most commonly used system is derived from Streptococcus pyogenes (Sp), which consists of a Cas9 endonuclease and two separate small RNAs, called tracrRNA and crRNA [13], that can be combined with a tetraloop to form a single guide RNA (sgRNA) [14]. SpCas9, which will be referred to 
henceforth as Cas9 for simplicity, cuts double strands of DNA to generate blunt-ended double strand breaks (DSBs) at 3 bp upstream of the NGG PAM (protospacer adjacent motif) under the guidance of sgRNA, which specifically recognizes the chromosomal loci of interest with 17-20 nucleotides (nt) $[15,16]$. Cells repair DSBs primarily by two mechanisms: non-homologous end joining (NHEJ) and homology-directed repair (HDR). In comparison to NHEJ, which generates a knockout phenotype by introducing variable insertions or deletions (indels) at the DSB, the HDR pathway creates precise deletions, base substitution, or insertion of coding sequences of interest in the presence of a recombination donor flanked with right and left homology arms (HA). Thus, the HDR pathway can be exploited to facilitate correction of diseased genes, insertion of epitope tags or fluorescent reporters, and overexpression of genes of interest in a site-specific manner.

Using rationally designed sgRNAs, high-level gene knockout can be achieved in different types of cells [1719]. However, improving the efficiency of precise CRISPR/Cas9-mediated gene editing or HDR-mediated knockin (KI) remains a major challenge, especially in human induced pluripotent stem cells and primary stem cells (iPSCs) [7, 20, 21]. Significant effort has been devoted to increasing knockin efficiency by improving targeting strategies, especially for insertion of a large DNA fragment. Previous reports used ZFN, TALEN, or CRISPR-Cas9 technology to knock in long DNA fragments via a homology-independent manner [22-26]. In these methods, the donor plasmid contains an endonuclease cleavage site and can be linearized in vivo when co-transfected with a specific endonuclease [22-26]. While these approaches are generic, they often lead to the integration of the entire donor plasmid and may induce mutagenic junctions caused by erroneous NHEJ, limiting the application potentials.

Hisano et al. [27] modified the donor plasmid by using short homologous sequences (20-40 bp) flanked by two sgRNA target sequences (also known as double cut donors), and observed efficient and precise integration of exogenous DNA into the predicted target locus in zebrafish. Similarly, donor vectors harboring microhomologous DNA ends have been used to edit human cells. However, the HDR efficiency before drug selection was not reported, which is likely to be less than 1\% [27-29]. Longer HA is necessary for efficient HDR in human cells. It has been reported that HDR of oligonucleotides is most efficient when a single-stranded oligodeoxynucleotide (ssODN) template with 90 nt HA is used [30]. For HDR knockin of a large fragment, HA of $\sim 0.2-0.8 \mathrm{~kb}$ have been reported to be necessary for transgene insertions and HA of up to $2 \mathrm{~kb}$ exhibited the optimal gene targeting efficiency in human iPSCs when a conventional circular donor is used [20].
However, no comprehensive studies have been reported on the comparison of the conventional circular plasmids and double cut donors in HDR efficiency and on the shortest HA that is required for high-level precise genome editing. Here we attempted to address these unanswered questions in $293 \mathrm{~T}$ cells and iPSCs. We further tested whether small molecules and other factors can increase HDR efficiency. We found that 20-30\% HDRmediated knockin can be achieved in human iPSCs using double cut donors with HA of $300-600$ bp in length together with cell cycle regulators Nocodazole and CCND1 (also known as cyclin D1).

\section{Results}

\section{A double cut HDR donor increases HDR efficiency in 293 T cells}

First, we used the most commonly used $293 \mathrm{~T}$ cells to compare the two donor plasmid designs and examine the effects of homology arm (HA) length on HDR efficiency. To this purpose, we established a reporter system in $293 \mathrm{~T}$ cells (Fig. 1a). First, we lentivirally transduced 293 T cells at a low multiplicity of infection (MOI of 0.1-0.2) with Lenti-EF1-Puro-sgRNA1-Wpre, which contains a sgRNA1 recognition sequence between Puro and Wpre element. After puromycin selection, we conducted single-cell cloning and generated three $293 \mathrm{~T}$ reporter lines. Following co-transfection with a promoterless mCherry donor plasmid and two plasmids encoding Cas9 and sgRNA1, mCherry is knocked into the target locus by HDR and the cells become mCherry ${ }^{+}$ (Fig. 1b). Although NHEJ insertion of donor may occur in this system, these cells would remain mCherry ${ }^{-}$. As such, the portion of $\mathrm{mCherry}^{+}$cells detected by flow cytometry (FACS) reflects HDR efficiency. We will address NHEJ integration in later sessions.

For correcting single nucleotide polymorphisms (SNPs) or generating precise point mutations, singlestranded oligodeoxynucleotides (ssODNs) have been successfully used as donor templates after creating a DSB [30-32]. For editing large pieces, doublestranded DNAs with relatively long HA are required to achieve relatively high efficiency [20]. The conventional donor is a circular plasmid carrying an insert flanked by two HA. Recently, polymerase chain reaction (PCR) products containing HA have also been used as donors due to its simplicity and lack of need for vector cloning [33, 34]. However, PCR products were similar to conventional donor plasmids in HDR efficiency, limiting its application potential (data not shown).

We hypothesize that in vivo cleavable donor plasmid can increase HDR; this can be achieved by sandwiching the donor vector with two sgRNA recognition sequences. When the Cas9/sgRNA complex surveys the genome and 


\section{a The mCherry HDR reporter system}
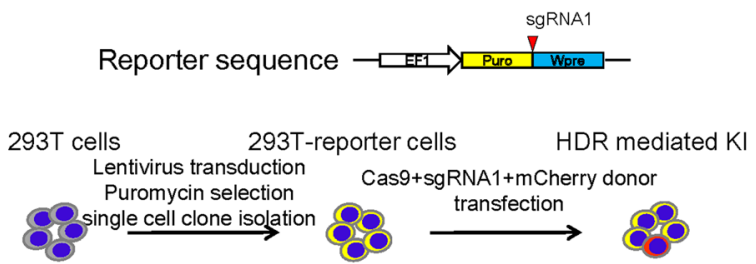

b Schematic of conventional vs. double cut donor

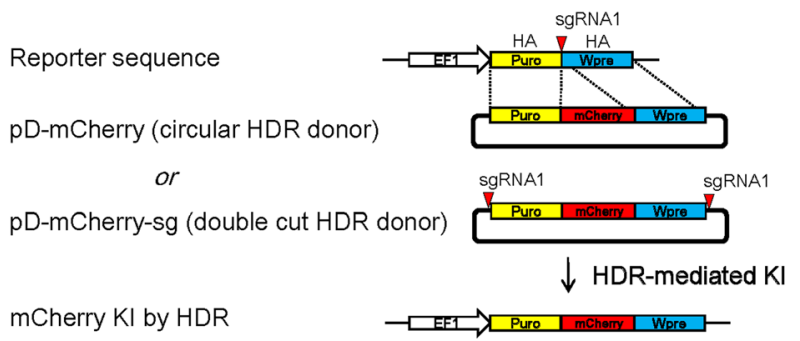

C Analysis of HDR efficiency by FACS 293T-reporter cells

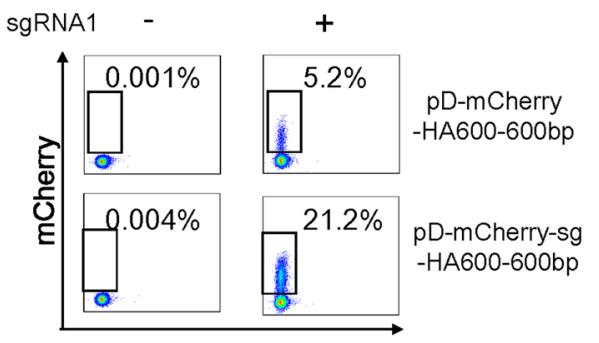

FL1 (irrelevant channel)

\section{d HDR efficiency by different donors}

293T-reporter cells

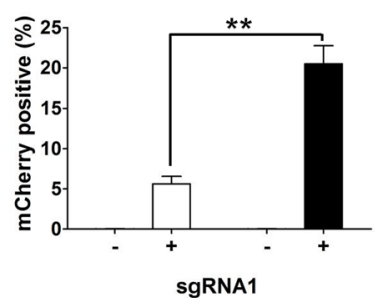

pD-mCherry-HA600-600bp pD-mCherry-sg-HA600-600bp

Fig. 1 A double cut HDR donor considerably increases HDR efficiency in 293 T cells after CRISPR-mediated DSB. a Schematic outline of the mCherry HDR reporter system. A lentiviral vector Lenti-EF1-Puro-sgRNA1-Wpre was used to generate reporter cell line. The red triangle indicates a sgRNA1-PAM sequence that will guide Cas9 to create DSB. 293 T cells were transduced with the lentiviral vector at a low MOI. After transduction, cells were treated with puromycin $(2 \mathrm{ug} / \mathrm{mL})$ and single-cell cloning was conducted to generate reporter cell lines with Puro-sgRNA1-Wpre target sequence (293 T reporter cells). EF1 is the promoter that drives the expression of a puromycin resistance gene. Wpre is the woodchuck hepatitis virus posttranscriptional regulatory element. After co-transfection with promoterless mCherry donor and two plasmids encoding Cas 9 and sgRNA1, the 293 T reporter cells use the donor to repair DSB by HDR pathway leading to the integration and expression of mCherry. $\mathbf{b}$ Design of promoterless mCherry HDR donors. pD-mCherry is a conventional circular HDR donor and pD-mCherry-sg is a double cut HDR donor in which the Puro-mCherry-Wpre cassette is flanked by two sgRNA1 recognition sequences. Puro (663 bp) and Wpre (592 bp) serve as left and right HA, respectively. To simplify naming scheme, the length of Puro and Wpre are unified as $600 \mathrm{bp}$ and the tag HA600-600 bp indicates their HA length. c FACS analysis of 293 T reporter cells one week after co-transfection of Cas9 and conventional vs. double cut pD-mCherry donors, with or without sgRNA1. The portions of mCherry ${ }^{+}$cells represent the HDR-mediated knockin efficiencies. $\mathbf{d}$ HDR efficiency by two different donors. $\mathrm{n}=3$; error bars represent S.E.M. Significance was calculated using the Student's paired t-test: ${ }^{* *} P \leq 0.01$

plasmids, it creates genomic DSB and linearizes donor plasmids simultaneously, thus synchronizing the demand and supply of homologous sequences and thereby increasing HDR. To test this idea, we compared the HDR efficiency using two types of donors: pD-mCherry, a conventional circular HDR donor and $\mathrm{pD}$-mCherry-sg, a double cut HDR donor in which the Puro-mCherry-Wpre cassette is flanked by two sgRNA1 recognition sequences
(Fig. 1b). In this manuscript, we tagged sg in the donor plasmid name to distinguish it from the commonly circular donor. In the two template plasmids, Puro (663 bp) and Wpre (592 bp) are identical and serve as left and right HA, respectively. To simplify the naming scheme, we unified the length of Puro and Wpre as $600 \mathrm{bp}$ and tagged a label of HA600-600 bp to the two donors to indicate their HA length. As expected, we observed a fourfold 
increase in the portion of mCherry ${ }^{+}$cells with double cut donor pD-mCherry-sg-HA600-600 bp compared to pDmCherry- HA600-600 bp (Fig. 1c, d).

\section{High HDR efficiency is achieved in 293 T cells by double cut HDR donors even with short homology arm}

For conventional HDR-mediated gene targeting, many investigators use $\mathrm{HA}$ lengths in the range of $0.1-2 \mathrm{~kb}$ for transgene insertions when a DSB is induced at the insertion site [20, 33, 35-42]. Having demonstrated the superior performance of double cut HDR donor in HDR, we decided to examine the effects of HA length on HDR efficiency. To this purpose, we designed a series of donors with HA in the range of 50-1500 bp in length. All of the double cut donors contain target sequence of sgRNA1 to flank the donor plasmids and can be linearized inside cells after co-transfection with Cas9 and sgRNA1 (Fig. 2a). As a control, we also designed a series of conventional circular HDR donors with various HA in the range of 300-1500 bp. We did not construct circular donors with shorter HA because HDR efficiency was as low as $0.22 \%$ when HA is $300 \mathrm{bp}$ (Fig. 2b, c and Additional file 1: Figure S1). When HA of the circular donors increased from 300 bp through 600-900 bp, HDR efficiency increased to $10 \%$ (Fig. 2b, $\mathrm{c}$ and Additional file 1: Figure S1).

Double cut donors increase the events of NHEJ [26], thus the donor with 0 bp HA (pD-mCherry-sg-HA0$0 \mathrm{bp)}$ was constructed to control the events of NHEJ. When $293 \mathrm{~T}$ cells were transfected with this donor, only $0.6 \%$ of cells expressed mCherry $\left(\mathrm{mCherry}^{+}\right)$, suggesting that NHEJ contributes only minimally to the percentage of mCherry $^{+}$cells (Fig. $2 b$ and Additional file 1: Figure S1). This result validates the use of percentage of mCherry ${ }^{+}$cells as an indicator of HDR efficiency. The HA as short as 50 bp led to a $6-10 \%$ HDR efficiency. With the increase of HA from 50 bp through 100$150 \mathrm{bp}$, a twofold increase in HDR efficiency was observed, suggesting that optimal HA length is at least $150 \mathrm{bp}$. A further increase of HA in double cut donors led to a gradual increase of HDR efficiency to $26 \%$ (Fig. 2b, c and Additional file 1: Figure S1).

Taken together, the above results conducted in $293 \mathrm{~T}$ cells suggest that a short HA of $300 \mathrm{bp}$ in circular donor is inefficient for HDR, whereas the same HA in double cut donor leads to significant HDR. The double cut donor system not only increases the HDR efficiency, but also reduces the demand for HA length.

\section{Enhanced HDR editing at the CTNNB1 locus in iPSCs with double cut HDR donors}

With promising results obtained in the $293 \mathrm{~T}$ reporter system, we attempted to edit a human iPSC line [43], because of its significance in regenerative medicine and well-known difficulty in editing human iPSCs in comparison to $293 \mathrm{~T}$ cells [26]. We first chose to target CTNNB1, a pivotal gene in the canonical WNT pathway that is constitutively expressed in iPSCs and other cells. We used a sgCTNNB1 to target $39 \mathrm{bp}$ before the stop codon (Fig. 3a), which showed a 60\% cleavage efficiency in iPSCs (Additional file 1: Figure S2). We then constructed a series of donors with GS-mNeonGreenWpre-polyA sequence being flanked by $\mathrm{HA}$ to this locus on both sides with various lengths (Fig. 3a, b). Silent mutations inside the gene were introduced to prevent cleavage in the middle of the donor by sgCTNNB1. GS is a quadruple GGGGS linker and mNeonGreen is a bright fluorescent protein [44]. HDR-mediated knockin leads to the formation of a CTNNB1-mNeonGreen fusion protein that fluoresces green. Similar to the above design, we constructed a series of circular donors (pDmNeonGreen) with HA in the range of 150-2000 bp and double cut donors (pD-mNeonGreen-sg) with HA of 50-2000 bp (Fig. 3b).

As the HA length of $\mathrm{pD}-\mathrm{mNeonGreen} \mathrm{donors} \mathrm{in-}$ creased from $150 \mathrm{bp}$ to $2000 \mathrm{bp}$, HDR efficiency at CTNNB1 progressively increased from $0.7 \%$ to $11 \%$ (Fig. 3b, c). In comparison, pD-mNeonGreen-sg donors showed 4-5\% HDR efficiency even with short HA of 50-100 bp. An increase of HA from $150 \mathrm{bp}$ through $300-600$ bp led a gradual increase of HDR from $8 \%$ to $12 \%$. However, further elongation of HA to $1000 \mathrm{bp}$, $1500 \mathrm{bp}$, or $2000 \mathrm{bp}$ in the double cut donors did not significantly increase HDR efficiency (Fig. 3b, c). Consistent with the notion that homologous recombination depends on HA, a donor with 0 bp HA (pD-mCherrysg-HA0-0 bp) showed 0\% HDR efficiency (Fig. 3b).

As double cut HDR donors have been used for NHEJmediated knockin [26], we determined NHEJ insertion events in this highly efficient genome editing system. Besides precise editing by HDR, there are two major possibilities of partial HDR and four possibilities of NHEJ insertions (Fig. 3d). To investigate these events in bulk iPSCs, we designed a pair of primers to specifically amplify the genomic locus without amplifying donors with HA of $50 \mathrm{bp}, 100 \mathrm{bp}, 150 \mathrm{bp}$, or $300 \mathrm{bp}$ (Additional file 1: Figure S3). The expected amplicon length is $824 \mathrm{bp}$ for wild-type allele and 2000-4000 bp for the edited allele (Fig. 3d). As expected, the first PCR gave a dominant $824 \mathrm{bp}$ band and weak bands of 2000-4000 bp, which were cut out for a second PCR. We purified and cloned the PCR product in the size of 2000-4000 bp into pJET vector and picked single bacterial colonies for Sanger sequencing (Fig. 3e).

We analyzed sequencing results from at least 30 clones per donor with quality data at both ends (Fig. 3f, Additional file 1: Figure S3). The majority of knockin events were HDR, with a $77 \%$ precise insertion rate 


\section{a}

\section{HDR donors with different length of homology arms}

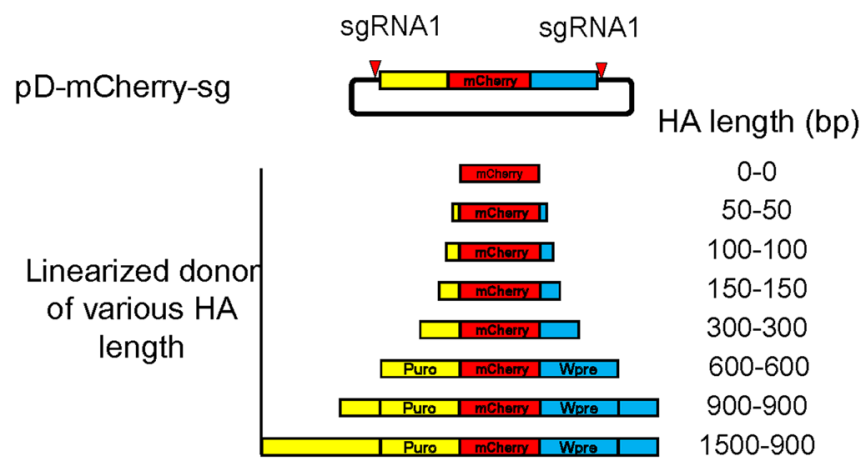

b Analysis of HDR efficiency by FACS

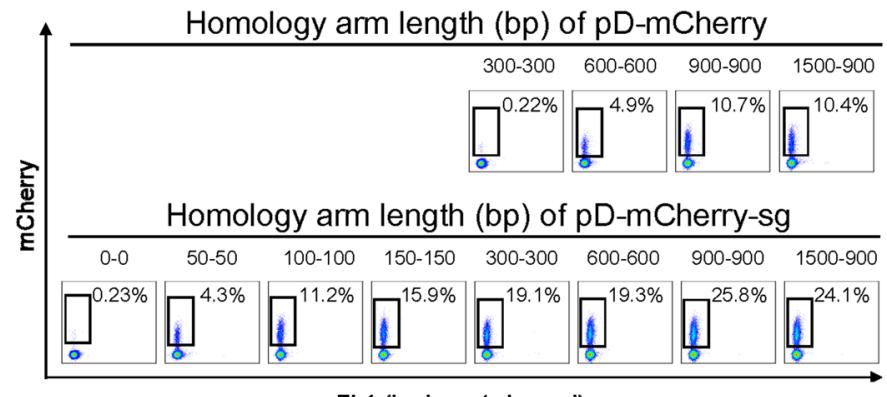

FL1 (irrelevant channel)

\section{HDR efficiency by different donors}

\section{T-reporter cells}

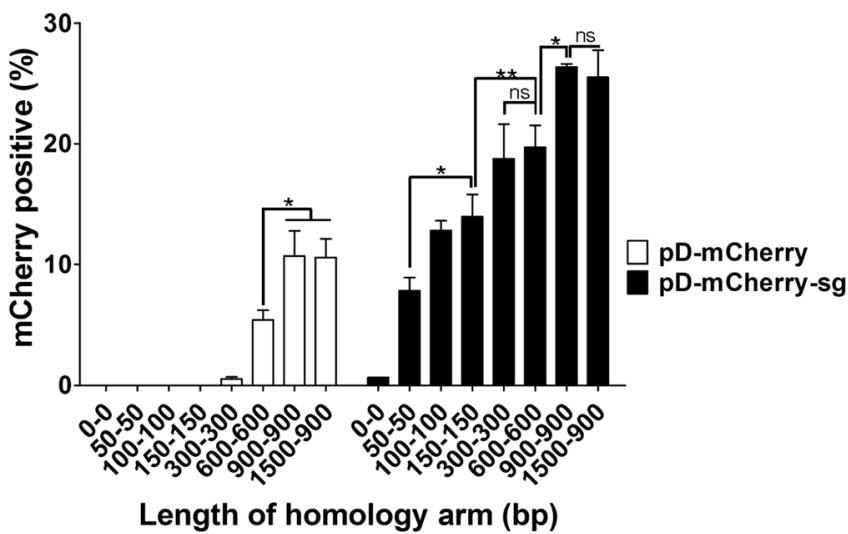

Fig. 2 High HDR efficiency is achieved in 293 T cells by double cut HDR donor even with short HA. a Schematic outline of pD-mCherry-sg (double cut HDR donor) with HA in the range of 0-1500 bp in length. The red triangle indicates a sgRNA target sequence. The left arm is marked as yellow and the right arm as blue. b Determination of the HDR efficiency by FACS. 293 T-reporter cells were analyzed one week after co-transfection of Cas9, sgRNA1, together with either pD-mCherry or pD-mCherry-sg. The percentages of mCherry ${ }^{+}$cells represent the HDR efficiencies. $\mathbf{c}$ Effects of HA length of conventional and double cut donors on HDR efficiency. $n=3$ biological replicates; error bars represent S.E.M. Significance was calculated using the Student's paired t-test: ${ }^{*} P \leq 0.05 ;{ }^{* *} P \leq 0.01 ;{ }^{* *} P \leq 0.001 ; n$ n not significant 
a Schematic of genome editing at the CTNNB1 locus

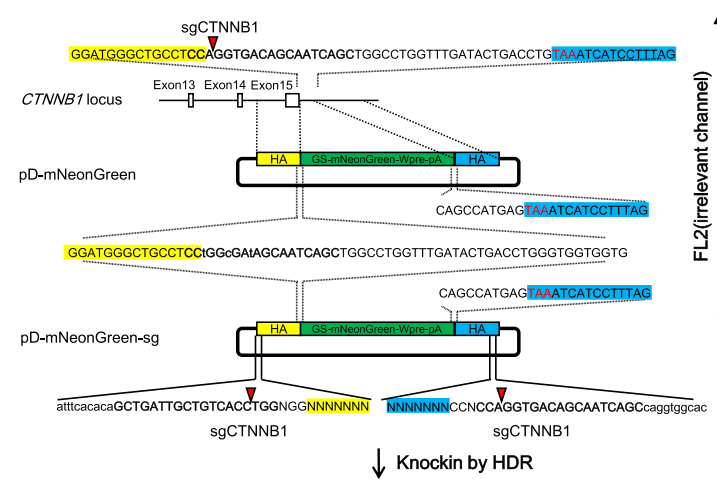

Precisely edited CTNNB1 locus

$$
\text { Exon13 Exon14 Exon15 }
$$

GGATGGGCTGCCTCCIGGCGAAAGCAATCAGCTGGCCTGGTTIGATACTGACCTGGGTGGTGGTG

\section{d Different knockin patterns}

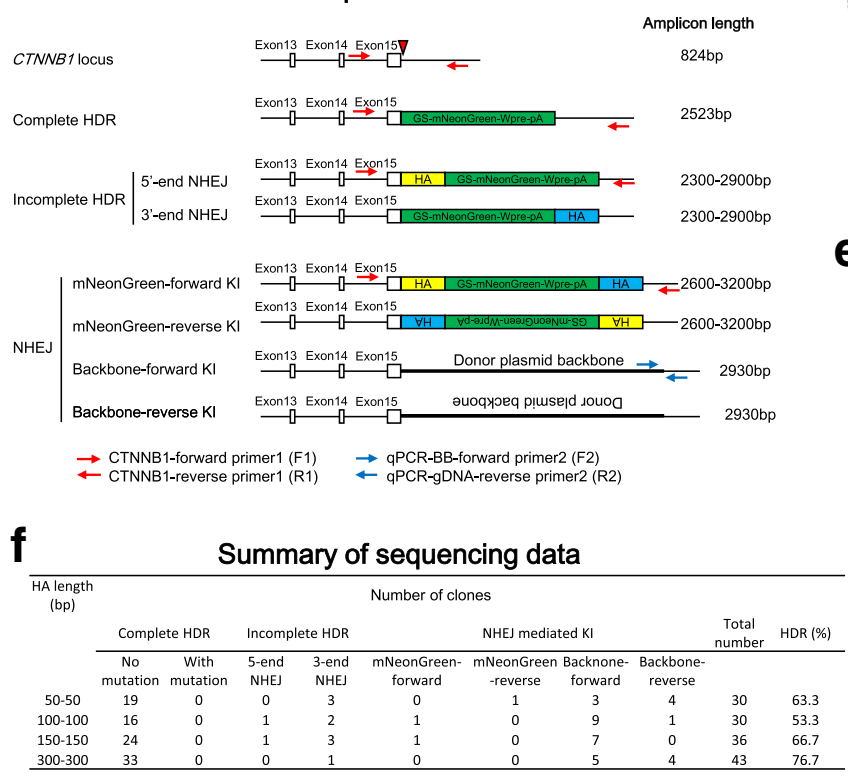

h Analysis of backbone insertion by qPCR

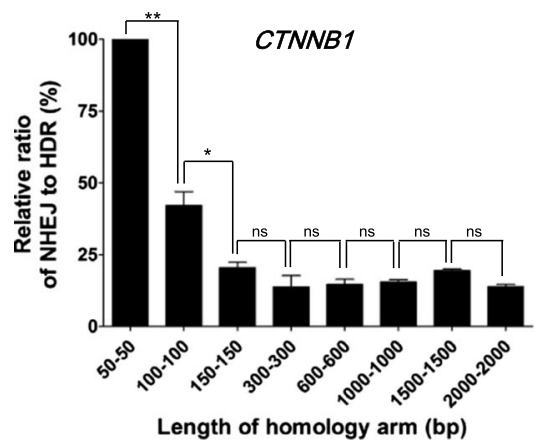

b Analysis of HDR efficiency by FACS

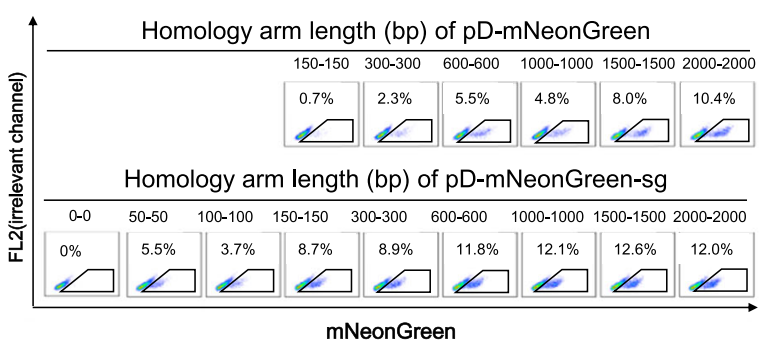

C HDR efficiency by donors with different HA length

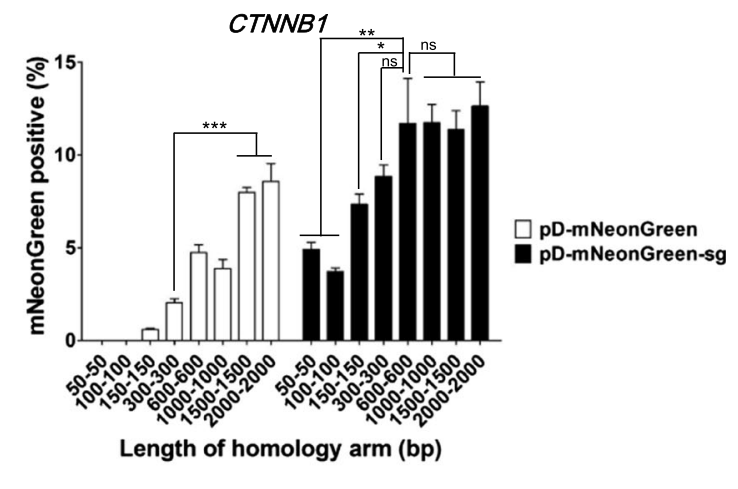

e Procedure for knockin pattern analysis

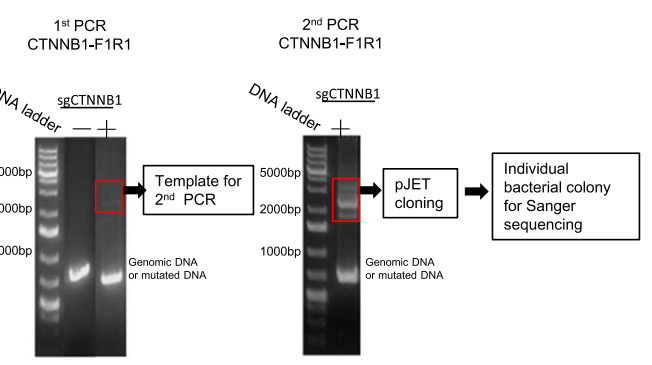

9 Knockin by HDR vs. NHEJ

CTNNB1

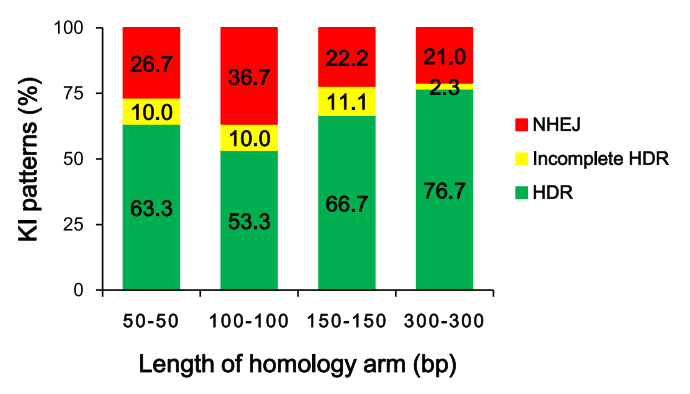

Fig. 3 (See legend on next page.) 
(See figure on previous page.)

Fig. 3 Genome editing in iPSCs at the CTNNB1 locus with conventional vs. double cut HDR donors of 50-2000 bp in HA length. a Schematic of genome editing at the CTNNB1 locus. The double strand break (DSB) is created by Cas9/sgCTNNB1 39 bp before the stop codon TAA (marked in red). The donors contain a GS-mNeonGreen-Wpre-polyA sequence sandwiched by left HA (yellow shadow) and right HA (blue shadow). GS is a linker. Silent mutations (lowercase and bold) were introduced to prevent cleavage by Cas9/sgCTNNB1. sgCTNNB1 sequence: bold; cut site: green triangle; stop codon: red; backbone: lowercase. $\mathbf{b}$ FACS analysis of iPSCs three days after nucleofection. The percentages of mNeonGreen ${ }^{+}$cells represent the HDR efficiencies. c Effects of HA length of conventional and double cut donors on HDR efficiency at the CTNNB1 locus. $n=4$. $\mathbf{d}$ Schematic of different knockin patterns. Apart from being edited by HDR, linearized insert sequence or backbone sequence can also integrate into the locus through incomplete HDR (HDR at one side and NHEJ at the other side) or NHEJ. A pair of primers (red arrows) was used to amplify edited sequence. The amplicon size is shown at the right side. For NHEJ knockin patterns, the length of PCR product is imprecise because NHEJ might be accompanied by indels. e Procedure for knockin pattern analysis. PCR was carried out for twice. The bands between the 2000-4000 bp area were cut off and cloned into pJET vector and individual bacterial colonies were picked for Sanger sequencing. f Summary of Sanger sequencing results. $\mathbf{g}$ Distribution of different knockin patterns by double cut HDR donors with different HA lengths. $\mathbf{h}$ Quantitative PCR (qPCR) analysis of donor plasmid backbone-forward insertion. $y$-axis indicates the relative ratio of NHEJ/HDR, in which NHEJ was calculated by qPCR data and HDR by the percentage of mNeonGreen ${ }^{+}$cells in a certain sample. Primers (F2 and R2) for qPCR analysis are indicated in blue in $(\mathbf{d}) . \mathbf{n}=3 . \mathbf{c}, \mathbf{h}$ Error bars represent S.E.M. ${ }^{*} P \leq 0.05 ;{ }^{* *} P \leq 0.01 ; n$ n not significant, by Student's paired t-test

being observed with HA of 300 bp (Fig. 3f, g). Due to limited numbers of clones that can be picked for Sanger sequencing, we decided to quantitate the relative NHEJ events by quantitative PCR (qPCR). We detected multiple forward backbone insertion (Fig. 3f), which can be used as a surrogate indicator to assess NHEJ. Primers were designed to specifically amplify this particular NHEJ event (Fig. 3d). The relative ratio of NHEJ/HDR was calculated by qPCR data (which is designed to amplify NHEJ insertion of plasmid backbone) divided by percentage of mNeonGreen $^{+}$cells (which reflects HDR insertion) in a certain sample. With the increase of HA from $50 \mathrm{bp}$ to $300 \mathrm{bp}$, the relative NHEJ was significantly dropped by $80 \%$, whereas further increase of the HA length did not lead to a further decrease in NHEJ (Fig. $3 \mathrm{~h}$ ), suggesting that a 300 bp homology on both arms of double cut donors is sufficient to increase HDR and/or suppress NHEJ.

\section{Reducing the length of replaced sequence surrounding DSB site enhances HDR}

The above study has achieved $77 \%$ precise editing in more than $10 \%$ of iPSCs. This HDR rate at the CTNNB1 site is lower than the PRDM14 site (see below). We noticed that, in the above donor design, sequences surrounding the Cas9/sgCTNNB1 cut site, $1 \mathrm{bp}$ in the left arm and $39 \mathrm{bp}$ on the right arm, need to be replaced before the sequence between the two HA on the donor can be inserted (Fig. 4a). After DSB formed, the sequence surrounding DSB will be used to search for the homologous sequence, thus the replaced sequence may be detrimental to HDR. We thus hypothesize that decreasing the length of replaced sequence will increase HDR and decrease NHEJ.

To test this hypothesis, we constructed another $\mathrm{pD}$ mNeonGreen-sg of $300 \mathrm{bp}$ homology. Compared to the former pD-mNeonGreen-sg-RS1-39 bp-HA300-300 bp (same as the donor pD-mNeonGreen-HA300-300 bp), the right $\mathrm{HA}$ in the new donor $\mathrm{pD}$-mNeonGreen-sgRS1-0 bp-HA300-300 bp extends to the cut site on genomic DNA, making the replaced sequence (RS) to be $0 \mathrm{bp}$ on the right side (Fig. $4 \mathrm{a}, \mathrm{b}$ ). As expected, the decrease of RS from 1-39 bp to 1-0 bp led to a $45 \%$ improvement in HDR efficiency (Fig. 4c), and knockin pattern analysis showed that the proportion of NHEJ decreased from $21 \%$ to $5 \%(P<0.05)$. Consequently, the HDR occurrence in bulk population increased from $77 \%$ to $88 \%$ (Fig. 4d, e, Additional file 1: Figure S4). In agreement with this result, qPCR that examines backbone insertion indicated a $\sim 40 \%$ decrease in the NHEJ/HDR ratio $(P<0.05)$ (Fig. $4 \mathrm{f})$.

To further validate this finding, we used $293 \mathrm{~T}$ reporter lines engineered with either a $50 \mathrm{bp}$ or $200 \mathrm{bp}$ sequence that need to be replaced on one or two arms before HDR. As expected, HDR rate was significantly decreased with RS of $200 \mathrm{bp}$ in one arm. When RS was present on both arms, an up to 50\% decrease in HDR was observed (Additional file 1: Figure S5). Taken together, these results suggest that in order to achieve high-level HDR and minimize NHEJ, two HA of the double cut donors should be identical to the sequences surrounding DSB.

\section{High HDR efficiency and low NHEJ occurrence at the PRDM14 locus by double cut HDR donor with short homology arms}

Due to heterogeneity of each locus, we asked whether the above results can be replicated in another site. We applied the same experimental strategy to another gene, PRDM14, a regulator of pluripotency [45]. An sgPRDM14 was designed to target the sequence surrounding the stop codon, with cleavage site at 4 bp downstream of the stop codon, and the cleavage efficiency of this sgPRDM14 was $\sim 30 \%$ in iPSCs (Additional file 1: Figure S2). We designed donors to in-frame insert 2A-GFP-Wpre-ployA sequence before the stop codon, 


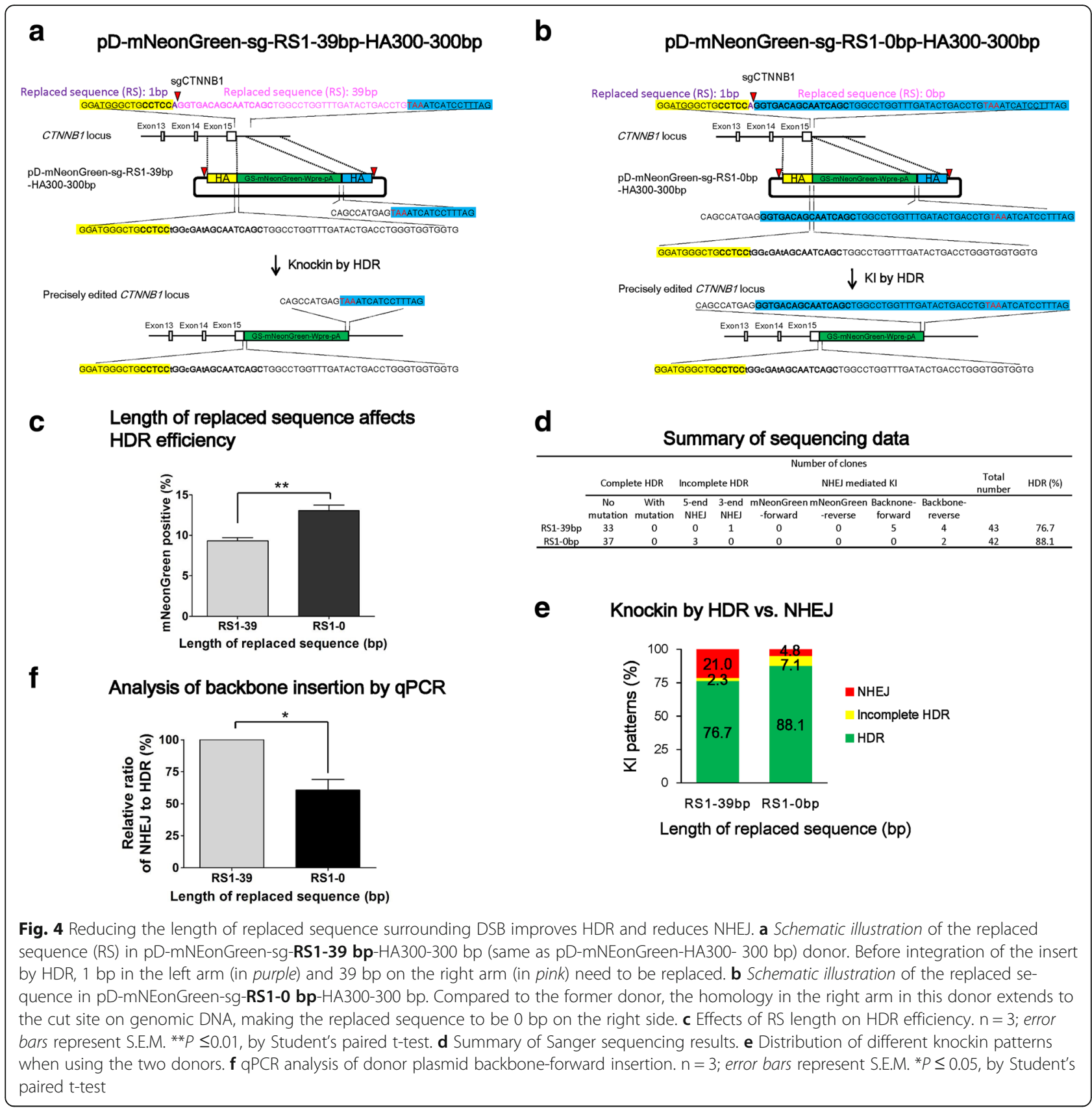

in which the 2A ribosome-skipping sequence allows for co-translation of PRDM14 and GFP. Similar to CTNNB1, we designed a series of circular HDR donors and double cut HDR donors with various HA length (Fig. 5a). A donor with 0 bp HA (pD-mCherry-sg-HA0$0 \mathrm{bp)}$ was used to control the events of NHEJ, which showed a $\sim 0 \%$ HDR efficiency (Fig. 5b). FACS analysis showed that HDR efficiencies have a tendency to increase along with the elongation of $\mathrm{HA}$ when using $\mathrm{pD}$ GFP, even though the general HDR efficiency was relatively low (1-3\%) (Fig. 5b, c). The pD-GFP-sg double cut donors showed a dramatic increase in HDR efficiency when HA length was extended from 50-100 bp (1-3\%) to $600 \mathrm{bp}(9 \%)$ (Fig. 5b, c).

Of interest, we observed that elongation of HA from $600 \mathrm{bp}$ to $1500 \mathrm{bp}$ or $2000 \mathrm{bp}$ led to a considerable decrease in HDR efficiency at the PRDM14 site (Fig. 5c), which is in contrast to the CTNNB1 site (Fig. 3c). This is not an artifact, because the same pattern was observed in independent studies conducted by two experimenters and the identities of the donor plasmids were doublechecked. This counterintuitive observation may be unique to this site and would be a subject for future investigation. 


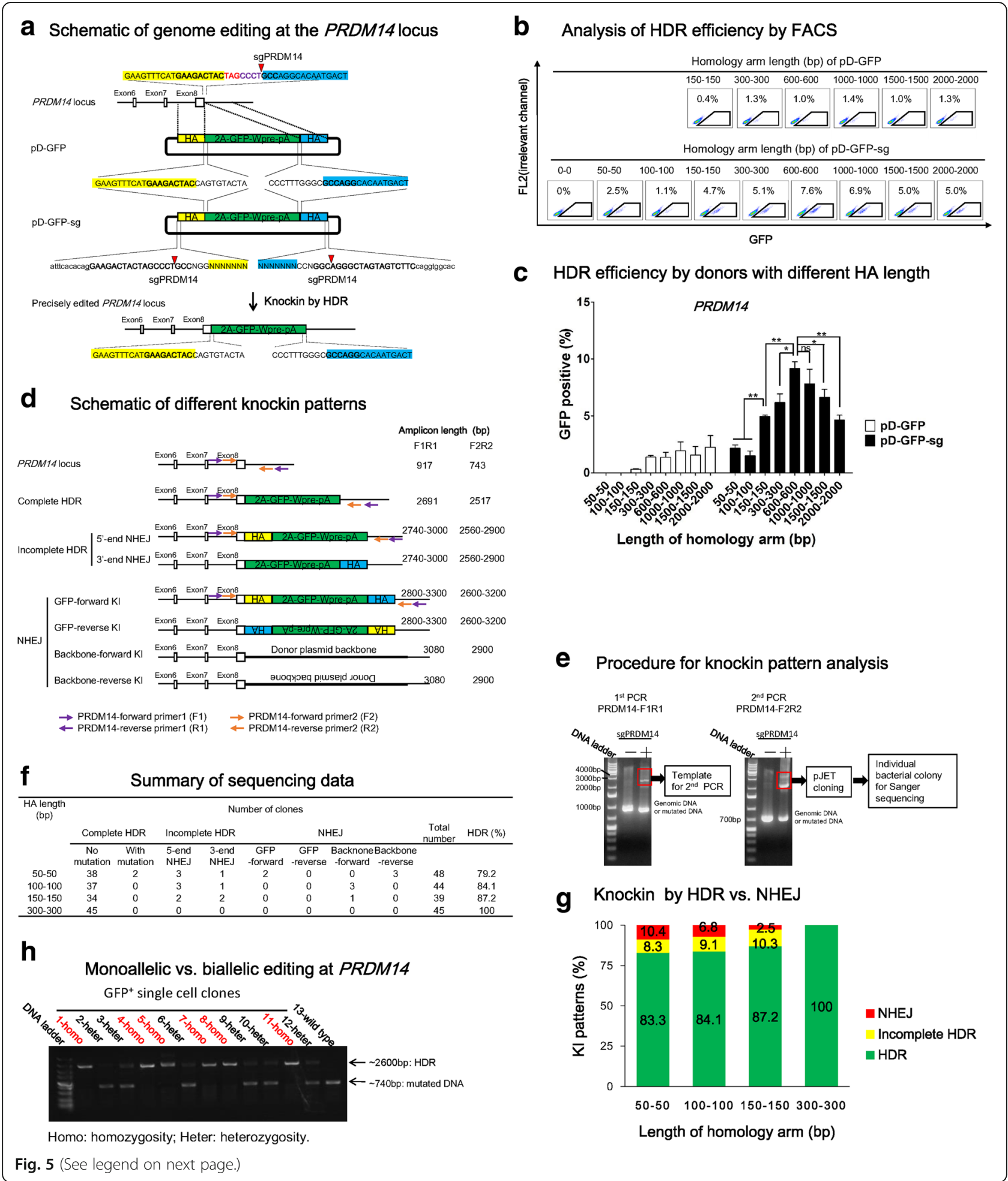




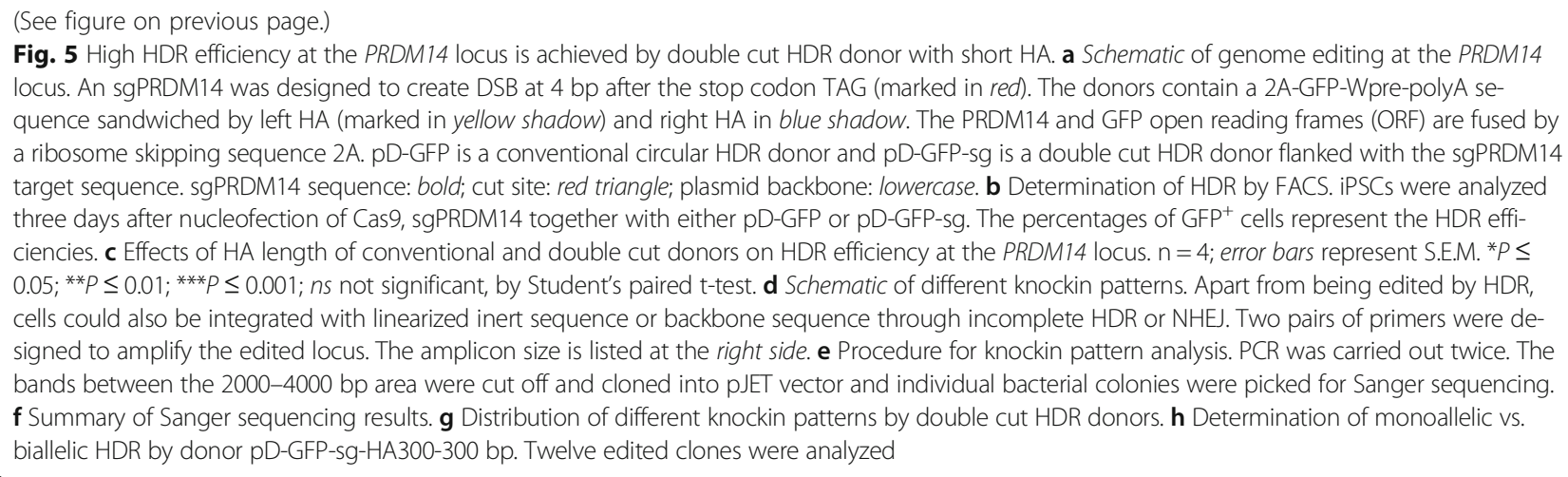

Similar to CTNNB1, we designed two pairs of primers to analyze knockin patterns of edited locus by donors with HA of $50 \mathrm{bp}, 100 \mathrm{bp}, 150 \mathrm{bp}$, or $300 \mathrm{bp}$ (Fig. 5d-f and Additional file 1: Figure S6). We obtained high quality sequencing data of $\sim 40$ clones for each donor plasmid and found that as HA length increased, from $50 \mathrm{bp}$ to $300 \mathrm{bp}$, the HDR rate increased from $80 \%$ to $100 \%$ (Fig. 5f, g). These data suggest that high-level HDR can be achieved with double cut donor, at least for locus like PRDM14.

We further examined the occurrence of biallelic HDR in iPSCs. We isolated $12 \mathrm{GFP}^{+}$single-cell clones by FACS sorting from the sample using pD-GFP-sgHA300-300 bp as the donor, as this condition provided high HDR efficiency and low NHEJ rate (Fig. 5f, g). PCR analysis showed one (homozygosity) or two (heterozygosity) bands with expected size. The 2600-bp band indicated amplification of HDR-edited allele. Among the random picked 12 clones, $50 \%$ showed biallelic HDR editing at the PRDM14 locus (Fig. $5 \mathrm{~h}$ ). In all the heterozygous clones, we observed indel mutations (Additional file 1: Figure S7).

\section{Double cut donor-mediated HDR can be further improved by cell cycle regulators}

Several small molecule compounds have been reported to significantly improve the CRISPR-mediated HDR efficiency through different mechanisms [20, 33, 46-48] (summarized in Additional file 1: Table S1). We asked whether these compounds can also increase HDR in our system. We tested multiple small molecules, including RS-1 (a stimulator of human homologous recombination protein RAD51) $[49,50]$, NU7441 (a DNA-PKcs inhibitor [46]), SCR7 (a DNA ligase IV inhibitor [20, 33, 49]), Brefeldin A [47], L755507 [47, 49], and Nocodazole [48]. To draw a solid conclusion, we examined the effects on HDR of both the CTNNB1 and PRDM14 locus using the verified highly efficient pD-sg-HA300-300 bp double cut donor. We added small molecules at their optimal concentration after nucleofection and changed the medium
$24 \mathrm{~h}$ later. HDR efficiency was analyzed on day 3 by FACS. RS-1, SCR7, and L755507 did not show significant improvement in HDR efficiency at both the PRDM14 and CTNNB loci, while Nu7441 and Brefeldin A showed a less pronounced improvement only at the CTNNB locus $(P<0.05)$. In contrast, treatment with Nocodazole, which synchronizes cell cycle at G2/M phase, increased HDR efficiency by $\sim 50 \%$ at both loci $(P<0.001)$ (Fig. 6a). Similar results were observed in $\mathrm{H} 1$ the human ES cell line (Additional file 1: Figure S8), demonstrating the reproducibility of this finding.

We also examined the effects of overexpression of RAD51, a key factor in the homologous recombination pathway, and Ad4E1B-E4orf6, which were reported to considerably increase HDR by inhibiting NHEJ [51]. In contrast to expectations, RAD51 or Ad4E1B-E4orf6 significantly decreased HDR efficiency in our system $(P<$ 0.001), at the two loci (Fig. 6b). Encouraged by the HDR-enhancing effects of Nocodazole, we tested CCND1, also known as cyclin D1, which induces cell cycle transition from G0/G1 to S-phase [52]. CCND1 showed a $\sim 20 \%$ improvement in HDR at both sites (Fig. 6b). We further applied Nocodazole and CCND1 together and found that they had an additive effect and increased HDR efficiency by $80-100 \%$ at both loci (Fig. 6c). This observation may be explained by that the combined use of CCND1 and Nocodazole increases cells in $\mathrm{S} / \mathrm{G} 2 / \mathrm{M}$ phases during which HDR is efficient, while they considerably decrease cells in G0/G1 phase during which double-stranded DNA (dsDNA) breaks are predominated repaired by NHEJ. As such, Nocodazole and CCND1 have an additive effect on enhancing precise genome editing.

Last, we asked whether increased HDR by CCND1 may lead to decreased NHEJ. We only tested the CTNNB1 locus, as using a 300 bp homology at PRDM14 already decreased NHEJ to a very low level. We used the optimized double cut donor pD-mNeonGreen-sg-RS10 bp-HA300-300 bp, which still showed an appreciable NHEJ rate. However, after co-transfection of iPSCs with 


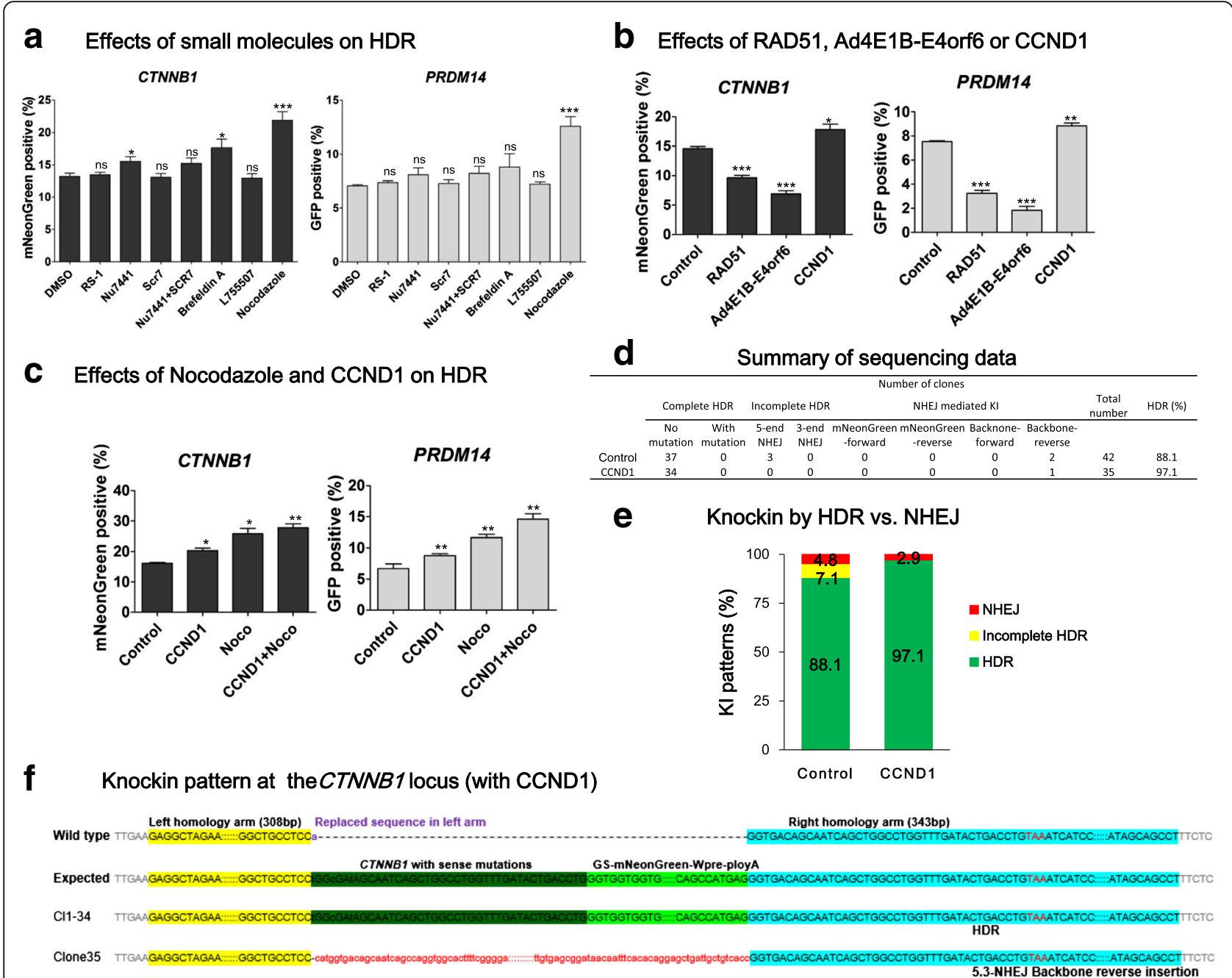

Fig. 6 Regulating cell cycle further improves HDR efficiency of the double cut donor system. a The effects of small molecules on HDR efficiency at the CTNNB1 or PRDM14 locus. The iPSCs were treated with RS-1 $(10 \mu \mathrm{M})$, Nu7441 $(2 \mu \mathrm{M})$, SCR7 $(1 \mu \mathrm{M})$, Brefeldin A $(0.1 \mu \mathrm{M}), \mathrm{L} 755507(5 \mu \mathrm{M})$, or Nocodazole $(100 \mathrm{ng} / \mathrm{mL})$ at 0-24 h after nucleofection and the HDR efficiency was determined by FACS on day 3. b The effects of RAD51, Ad4E1B-Eorf46, and CCND1 on HDR efficiency at the CTNNB1 or PRDM14 locus. The plasmid encoding RAD51, Ad4E1B-Eorf46, or CCND1 was cotransfected with Cas9, sgRNA, and pDonor. The HDR efficiency was examined by FACS on day 3. c The effects of Nocodazole and CCND1 on HDR efficiency at the CTNNB1 or PRDM14 locus. The plasmid encoding CCND1 was co-transfected with Cas9, sgRNA, and donor plasmid. Nocodazole $(100 \mathrm{ng} / \mathrm{mL})$ was added into the medium at 0-24 h after transfection. HDR efficiency was determined by FACS on day 3. a-c Comparison with control by Student's paired t-test: ${ }^{*} P \leq 0.05 ;{ }^{* *} P \leq 0.01 ;{ }^{* * *} P \leq 0.001$; $n$ s not significant. $\mathbf{d}$-f CCND1 increases HDR rate at the CTNNB1 locus. The procedure for knockin pattern analysis was detailed above. At least 30 colonies were picked for Sanger sequencing at both ends

CCND1 and HDR plasmids, the NHEJ events were decreased from $12 \%$ to $3 \%$ (Fig. $6 \mathrm{~d}-\mathrm{f}$ ). These data suggest that cell cycle regulators not only increase HDR but also suppress NHEJ.

High HDR efficiency in human iPSCs using this approach will find its way to diverse applications. Take one, after tagging a gene of interest with a flexible G4S linker and a fluorescent protein gene, a fusion protein will be formed, which can be used to quantitate the gene expression level by FACS and map its cellular localization by confocal microscopy. Here we targeted mNeonGreen to the stop codon of CTNNB1 and $t d T o-$ mato to the stop codon of PRDM14 and examined the cells by confocal microscopy. As expected, the PRDM14-tdTomato fusion protein was localized in the nucleus (Additional file 1: Figure S9a). Of interest, we observed the mNeonGreen signal on plasma membrane, indicating that CTNNB1 is largely localized at iPSC membrane (Additional file 1: Figure S9b).

\section{Discussion}

Here we report that a double cut donor vector design together with the cell cycle regulators leads to high-level HDR-mediated precise integration of a 2-kb piece of DNA in iPSCs. We show that the double cut donor with a targeting cassette flanked by two sgRNA recognition 
sequences leads to a twofold to fivefold increase in HDR efficiency compared to the circular donor plasmid. The optimal HA length of double cut donors for achieving high-level HDR is $600 \mathrm{bp}$ and further elongation only slightly increases or even decreases HDR at different sites. Minimizing the replaced sequence surrounding the DSB increases HDR and suppresses NHEJ-mediated insertion. The combined use of cell cycle regulators Nocodazole and CCND1 leads to an additional 100\% increase in HDR efficiency. After optimization, less than 3\% NHEJ-mediated insertion of donor plasmid sequence can be detected. These findings should have important implications for precision medicine.

The length of HA is the most critical factor for targeting template design. For precise editing of point mutations or SNPs, ssODNs are often used as a DNA template and homology of 30-70 bases on either arm is sufficient for high-level HDR [30, 53]. However, precise editing, in particular insertion of a large piece, remains inefficient in cells of significant research and clinical interest, such as human pluripotent stem cells. In early gene targeting studies, the homology of the two arms was recommended to be $5-8 \mathrm{~kb}$ in total and more than $1 \mathrm{~kb}$ for the shorter arm. After the advent of artificial nuclease and CRISPR technologies, HA lengths ranging from $100 \mathrm{bp}$ to several $\mathrm{kbp}$ have been used for successful gene editing [20,33, 40-42]. The optimal HA length is reported to be $2 \mathrm{~kb}$, at least in human iPSCs [20]. We reasoned that simultaneous cleavage of the genomic and plasmid DNA makes the HA more accessible, thus the optimal HA might be shorter. To identify the optimal HA length, we evaluated the homology length in the range of $50-2000 \mathrm{bp}$ at two to three distinct loci. The shortest length we tested is $50 \mathrm{bp}$, because $40-50$ bp HA have been previously used by other investigators [27, 34, 54].

Using stringent promoterless donor HDR reporter systems, we systematically compared conventional circular HDR donors with double cut HDR donors. We constructed donors to insert florescent transgenes into each target locus, so that HDR events can be detected by FACS after precise transgene knockin. Since HDR efficiency differs from one site to another, we studied three sites. We observed that the effects of HA length on HDR efficiency is not identical for the three sites. However, careful examination of our data leads to several conclusions. First, for conventional circular HDR donor, a HA of less than $150 \mathrm{bp}$ is insufficient to guide precise genome editing. An increase of HA from $300 \mathrm{bp}$ to $2000 \mathrm{bp}$ shows a trend of continued increase in HDR efficiency. This is consistent with a previous report showing an optimal HA length of $2000 \mathrm{bp}$ in iPSCs [20]. Second, HA as short as $50 \mathrm{bp}$ in the double cut donor design lead to appreciable HDR, whereas elongation of
HA to 150 bp considerably increases HDR efficiency. The highest HDR is achieved when HA is $600-1000$ bp in length. Third, the use of double cut donor increases HDR efficiency by twofold to fivefold compared to circular plasmid donor. We noted that the optimal HA length is very similar to the DNA length dependence of the single-strand annealing (SSA) pathway in Saccharomyces cerevisiae, the budding yeast [55], suggesting that the DSB in our system is most probably repaired by the SSA pathway. This may also explain why most HA enhancers and NHEJ inhibitors do not show positive effects on HDR in our study.

Taking all factors into consideration, we recommend the use of $600 \mathrm{bp} \mathrm{HA}$ for double cut donor vector design, for the following reasons. First, $600 \mathrm{bp}$ is the optimal length for some sites and further increases in HA length only slightly enhances HDR at some locus, while a HA with more than 1000 bp may be even detrimental for some locus. Second, construction of donor vector with two $600 \mathrm{bp}$ homology arms is an economical choice, because PCR amplification of short pieces from the genomic DNA (gDNA) template is very efficient. In addition, the vector verification can be easily conducted by Sanger sequencing, which usually provides quality data of $700-800 \mathrm{bp}$.

In our study, we used one sgRNA to target both gDNA and the donor plasmid. In previous studies, two sgRNAs were used, one for creating genomic DSB and another for releasing donor template from the plasmid [26, 27]. This design increases complexity and occasionally may not be able to perfectly synchronize the demand and supply of homologous sequences, because the cleavage efficiencies of the two distinct sgRNAs may not be identical. In support of this hypothesis, we found that the use of two sgRNAs in the double cut donor system decreases HDR by $\sim 10 \%$, albeit no significant difference is achieved (Additional file 1: Figure S10).

Another consideration for donor template design is the distance of the insertion locus from the DSB, which should be as short as possible and 100-200 bp away, might decrease the knockin efficiency by $\sim 80 \%$ when a conventional donor plasmid is used [37]. In the double cut donor system, a 50-100 bp replaced sequence leads to a $50 \%$ decrease in HDR efficiency in $293 \mathrm{~T}$ cells (Additional file 1: Figure S5). Similarly, a 39 bp replaced sequence at the CTNNB1 locus induces a $30 \%$ decrease in HDR in iPSCs (Fig. 4c). As such, the ideal donor should be designed to have two HA identical to sequences surrounding DSB created by Cas9/sgRNA.

We also investigated whether commonly used HDR boosters can improve knockin efficiency in our system. Thus far, several compounds have been reported to be able to improve knockin efficiency. Suppression of the NHEJ key enzymes such as DNA Ligase or DNA PK has 
been shown to stimulate Cas9-mediated HDR at the expense of NHEJ [20,33, 46]. NU7441, an inhibitor of DNA-PKcs, increases the knockin efficiency by twofold in $293 \mathrm{~T}$ cells [46]. SCR7, an inhibitor of DNA ligase IV, improves the knockin efficiency by twofold to 19-fold in two reports [20, 33]. Brefeldin A and L755507 increase HDR twofold to threefold in mouse pluripotent stem cells [47]. In addition, RS-1, which upregulates homologous recombination factor RAD51 [56], increases nuclease-mediated knockin efficiency by twofold to fivefold $[49,50]$. In contrast to these reports, none of these molecules consistently enhances HDRs at both CTNNB1 and PRDM14 loci in human iPSCs. In further support of these results, we found that overexpression of RAD51 or Ad4E1B-E4orf6, which degrade ligase IV, is detrimental to HDR (Fig. 6b). One explanation for the discrepancy between our results and the published data is that our optimized vector has achieved high-level HDR, which masks the subtle changes mediated by many inhibitors. Alternatively, the effects of these factors are cell line dependent. In support of this notion, unimpressive effects of SCR7 and L755507 were also reported in recent publications using different cell lines or cells from different species $[49,50]$.

Another strategy to enhance HDR takes advantage of the fact that NHEJ occurs during the G1, S, and G2 phases, whereas HDR-mediated repair is restricted to the late $\mathrm{S}$ and $\mathrm{G} 2$ phases $[57,58]$. Timed delivery of Cas9 protein and sgRNAs into cells arrested at the G2/ $M$ phase after Nocodazole treatment significantly increases HDR events [48]. We found that cell cycle synchronization with Nocodazole in our plasmid Cas9/ sgRNA system can increase HDR efficiency by $~ 50 \%$ (Fig. 6a and Additional file 1: Figure S8). In addition, CCND1 increases HDR by $20 \%$, which may take effect by pushing cells at $\mathrm{G} 1$ phase into the $\mathrm{S}$ phase. Moreover, Nocodazole synergizes with CCND1 to increase HDR efficiency by $\sim 100 \%$. The additive effect is due to Nocodazole synchronizing the cell cycle at the G2/M phase and to CCND1 inducing cell cycle transition from the G0/ G1 to the $S$ phase, leading to more cells in the S/G2/M phases during which dsDNA breaks are preferentially repaired by HDR instead of NHEJ. Similar strategies have been used to increase HDR and decrease NHEJ incidence by creating a fusion protein of Cas 9 and a peptide from Geminin, which is expressed during S/G2/M and degraded during G1 $[59,60]$. However, this approach may occasionally decrease HDR due to decreased Cas9 protein levels. The use of CCND1 is preferable, because it enhances HDR as well as decreases NHEJ insertion. Alternatively, integration of all these strategies may lead to enhanced precise genome editing.

HDR efficiency in human pluripotent stem cells, including iPSCs or embryonic stem cells, is often much lower than other types of cells [61]. Approximately 1\% or lower HDR efficiency is generally achieved after inducing DSB [21, 42, 62, 63], although an up to $11 \%$ efficiency has been reported, which might be cell line dependent and is limited to homozygous gene replacement [20]. Using the double cut HDR donor, we achieved $\sim 14 \%$ HDR at CTNNB1 and $~ 10 \%$ at PRDM14. Combined use of Nocodazole and CCND1 further increases the HDR efficiency to $20-30 \%$. Given that iPSCs have been widely used for disease modeling and regenerative medicine, this improvement will ease the burden for identification of correctly edited cells by single-cell cloning and sequencing.

Recently, ribonucleoproteins (RNPs), the Cas9 protein in complex with in vitro transcribed guide RNA, have been used together with a single-stranded oligonucleotide HDR template for nucleotide replacement or insertion [48, 64, 65]. Moreover, chemically modified guide RNAs can further enhance genome editing efficiency [66]. We believe that the double cut donor vector can also be delivered together with Cas9 protein/RNA and sgRNA transcripts, which may even be preferable in applications such as zygote injection for creating knockin animals.

\section{Conclusion}

In summary, synchronizing the demand and supply of homologous sequences, by flanking the targeting vector with two sgRNA recognition sites that are identical to the sgRNA target site on the genome, leads to a twofold to fivefold increase in HDR-mediated knockin. The double cut donor template design requires HA as short as $600 \mathrm{bp}$ to enable high-level precise insertion of a large piece of DNA. We believe that the improved targeting strategies are broadly applicable in generating precise knockin or reporter animals and human cell lines for basic research and disease modeling. Further improvements of the double cut donor system may contribute to the success of next-generation clinical gene therapy.

\section{Methods}

\section{Lentiviral vector construction}

The complementary DNA (cDNA) for a puromycin resistant gene (Puro) was amplified by PCR and purified using KAPA HiFi polymerase (KAPA Biosystems) and a GeneJET Gel Extraction Kit (Thermo Fisher Scientific), respectively. The open reading frame of the Puro gene was inserted into a lentiviral vector with the EF1 promoter, used to drive the expression of Puro. The sgGFP target sequence together with the NGG PAM (GGTGCAGATGAACTTCAGGG) was in-frame PCRcloned into the vector by incorporating the relevant sequences in the primers. To construct vectors with the GFP gene between the Puro and Wpre element, while 
still harboring sequences surrounding DSB that are nonhomologous to the targeting vector, a $\sim 50$ bp or $\sim 200$ bp irrelevant sequence was introduced between the sgRNA1 recognition sequence and the Puro and/or Wpre element. Multiple gene inserts were cloned into lentiviral vector backbones using the NEBuilder HiFi DNA Assembly Kit (New England Biolabs), following manufacturer's instructions. All constructs were verified by Sanger sequencing (MCLAB). Correct clones were grown in CircleGrow Media (MP Biomedicals) and DNA plasmids were purified using Endo-Free Plasmid Maxi Kits (Qiagen). A standard calcium phosphate precipitation protocol was used for lentivirus production as previously described [32]. The lentiviral vectors were concentrated a 100-fold by centrifugation at $6000 \mathrm{~g}$ for 24 h at $4{ }^{\circ} \mathrm{C}$ to reach titers of $2-10 \times 10^{7} / \mathrm{mL}$.

\section{GFP reporter cell line}

Human embryonic kidney (HEK) $293 \mathrm{~T}$ cells were transduced with lentiviral vectors (Lenti-EF1-Puro-GFP-Wpre, Lenti EF1-Puro-sgRNA1-Wpre; Lenti EF1-Puro-GFP(nh0200 bp)-Wpre, Lenti EF1-Puro-GFP(nh200-0 bp)-Wpre, Lenti EF1-Puro-GFP(nh50-50 bp)-Wpre, and Lenti EF1Puro-GFP(nh200-200 bp)-Wpre) at a low MOI of 0.1-0.2, and stably transduced cells were selected for by supplementing culture medium with $1 \mu \mathrm{g} / \mathrm{mL}$ puromycin. After one week of antibiotic selection, cell lines expressing puromycin resistance and either high GFP ( $>98 \%)$ or no GFP production (in cases of GFP fragments or no GFP insert) were established.

\section{sgRNA design}

The CHOPCHOP website (https://chopchop.rc.fas.harvard. edu/) was used to design high-performance sgRNAs targeting various positions on GFP sequence and the stop codons of the human CTNNB1 and the human PRDM14 genes [67]. We preferentially chose sgRNAs with a $\mathrm{G}$ at the $5^{\prime}$ end which initiates U6-promoter-mediated transcription [16]. Five sgRNAs were used in this study: sgRNA1 (GGTGCAGATGAACTTCA), sgRNA2 (GCTAGTGGGG TTGATAGGAG), sgRNA3 (GCCGGGAGCAGGCGTGAGTG), sgCTNNB1 (GCTGATTGCTGTCACCTGG), sgPRDM14 (GAAGACTACTAGCCCTGCC), among which sgRNA1, sgRNA2, and sgRNA3 do not target human genome.

\section{Cas9 and sgRNA plasmid construction}

All Cas9 and sgRNA plasmids were constructed with a NEBuilder HiFi DNA Assembly Kit (New England Biolabs). First, PCR products were produced using KAPA HiFi polymerase (KAPA Biosystems) and purified using a GeneJET Gel Extraction Kit. The linear PCR products were then assembled into plasmids in a DNA assembly reaction $(20 \mathrm{uL})$, on ice, according to the manufacturer's instructions. The reaction contained NEBuilder HiFi DNA Assembly Master Mix (10 uL), equal ratios of PCR products $(0.2-0.5 \mathrm{pmols})$, and deionized water. The ligation reaction was briefly vortexed and centrifuged prior to incubation at $50{ }^{\circ} \mathrm{C}$ for $5-30 \mathrm{~min}$. NEB 5-alpha Competent E. coli cells were then transformed with the assembled DNA products and plated on ampicillintreated agar plates. Multiple colonies were chosen for Sanger sequencing (MCLAB) to identify the correct clones using the primer U6-F: GGGCAGGAAGAG GGCCTAT.

\section{Donor plasmid construction}

All of the donor plasmids used in this study were generated with a CloneJET PCR Cloning Kit (Thermo Scientific). To construct pJET donor plasmids, the homology repair templates were amplified by PCR using KAPA HiFi polymerase (KAPA Biosystems) and purified using a GeneJET Gel Extraction Kit. To clone donor plasmids harboring sgRNA recognition sites, the sgRNA target sequence together with a PAM (NGG) was included in both the forward and the reverse primers. A ligation reaction $(20 \mathrm{uL})$ was performed, on ice, according to the manufacturer's instructions, containing $2 \mathrm{X}$ Reaction Buffer $(10 \mathrm{uL}), \mathrm{pJET} 1.2 /$ blunt Cloning Vector $(50 \mathrm{ng} / \mu \mathrm{L})(1$ $\mathrm{uL})$, T4 DNA Ligase $(1 \mathrm{uL})$, purified PCR product $(0.15$ pmol), and nuclease-free water (remaining volume). The ligation reaction was then briefly vortexed and centrifuged prior to incubation at room temperature $\left(22{ }^{\circ} \mathrm{C}\right)$ for 5-30 min. NEB 5-alpha Competent $E$. coli cells were then transformed with the ligation product and plated on ampicillin-treated agar plates. Multiple colonies were chosen for Sanger sequencing (MCLAB) to identify the correct clones using the primers pJET1.2-F: CGACT CACTATAGGGAGAGCGGC and pJET1.2-R: AAGAA CATCGATTTTCCATGGCAG. Correct clones were cultured and DNA plasmids were purified, as previously described. To construct donor plasmid targeting CTNNB1 at 39 bp before the stop codon, the left and right HA were amplified from human genomic DNA; with the stop codon being removed and in-frame linked with the GS sequence (a quadruple of GGGGS peptides); the insert mNeonGreen-Wpre-polyA was amplified from another vector in the lab. A sgCTNNB1 target sequence together with the PAM sequence (GCTGATTGCTGT CACCTGGAGG) was tagged at a location outside of the two HA. To construct donor plasmids targeting the PRDM14 stop codon, the left and right HA were amplified from human genomic DNA, with the stop codon being removed and in-frame linked with the $2 \mathrm{~A}$ sequence; the insert 2A-GFP-Wpre-polyA was amplified from another vector in the lab. A sgPRDM14 target sequence together with the PAM sequence (GGAAGACTACTAGCCCTGCCAGG) was tagged to the regions 
flanking the upstream and downstream HA. The CTNNB1 and PRDM14 donor template plasmids were generated with a NEBuilder HiFi DNA Assembly kit (New England Biolabs), as previously described.

\section{Construction of putative HDR-enhancing plasmids}

To test if extra factors can enhance double cut donor mediated HDR, we examined RAD51, CCND1 (Cyclin D1), and Ad4 E1B55K and E4orf6 [51]; the latter two genes were linked together using a ribosome-skipping E2A sequence. cDNAs for RAD51 and CCND1 were purchased from DNASU, and Ad4 E1B and E4orf6 were purchased from Addgene (Plasmid \#64218 and 64222). The EF1 promoter was used to drive the expression of these genes and the Wpre-polyA cassette was tagged downstream of the stop codon to increase transgene expression levels. All vectors were confirmed by sequencing.

\section{Cell culture}

HEK 293 T cells were cultured in DMEM (Dulbecco's modified Eagle medium) supplemented with $10 \%$ fetal bovine serum (FBS; $A B M$ ) and $1 \%$ penicillin/streptomycin. Human iPSCs used in this study were generated from peripheral blood mononuclear cells after lentiviral transduction of five reprogramming factors [43]. Feederfree iPSCs were maintained on Matrigel-coated plates (BD) and cultured in mTeSR1 (Stemcell Technologies) according to the manufacturer's instructions. The ROCK inhibitor Y-27632 $(10 \mu \mathrm{M}$, Millipore $)$ was added to the culture medium before, during, and after, passaging with Accutase. All cells were cultured at $37{ }^{\circ} \mathrm{C}$ with $5 \% \mathrm{CO}_{2}$.

\section{Transfections}

For transfection of HEK 293 T cells, Lipofectamine 3000 (Life Technologies) was used according to manufacturer's instructions. For transfection of human iPSCs, cells were electroporated using the Lonza Nucleofector system (Lonza). The Human Stem Cell Nucleofector ${ }^{\bullet}$ Kit 2 and the program B016 were used as outlined in the manufacturer's instructions. For nucleofection, $1 \mu \mathrm{g}$ of each plasmid was used.

\section{T7E1 cleavage assay}

iPSCs were nucleofected with either sgCTNNB1 or sgPRDM14 together with Cas9 plasmid. Three days later, genomic DNA was isolated using the DNeasy Blood \& Tissue Kit (Qiagen) according to the manufacturer's instructions. For cells targeted with sgCTNNB1, the predicted flanking sequence was PCR-amplified using CTNNB1-F1 and CTNNB1-R1 primers; for cells treated with sgPRDM14, PRDM14-F2 and PRDM14-R2 primers were used. The amplicons were denatured by heating and annealed to form heteroduplex DNA, which was treated with 5 units of T7 endonuclease 1 (New England
Biolabs) for $20 \mathrm{~min}$ at $37{ }^{\circ} \mathrm{C}$ and then analyzed by $2 \%$ agarose gel electrophoresis. The cleavage frequency was calculated from the proportion of cut bands intensity to total bands intensity.

\section{Small molecules}

To test the effect of small molecule compounds, iPSCs were evenly split into eight wells after nucleofection with Cas9/sgRNA and the relevant double cut donor. DMSO control $(0.1 \%), \operatorname{RS}-1(10 \mu \mathrm{M}), \operatorname{Nu} 7441(2 \mu \mathrm{M})$, SCR7 $(1 \mu \mathrm{M})$, Brefeldin A $(0.1 \mu \mathrm{M})$, L755507 $(5 \mu \mathrm{M})$, Nocodazole $(100 \mathrm{ng} / \mathrm{mL})$, or Nu7441 $(2 \mu \mathrm{M})$ and SCR7 $(1 \mu \mathrm{M})$ were added in the wells for the first $24 \mathrm{~h}$ and then the medium was changed with fresh medium thereafter. Three days after nucleofection, cells were harvested for FACS analysis to determine the HDR efficiency in each condition.

\section{Flow cytometry}

To determine the percentage of cells that are mCherrypositive, mNeonGreen-positive, or GFP-positive (Knockin by HDR), cells were disassociated with Accutase and analyzed on a BD FACSAria III flow cytometer. Cells were first gated for the intact cell population using forward scatter versus side scatter plots and then gated for single cells based on forward scatter W versus forward scatter $\mathrm{H}$. The means and standard errors from at least three independent experiments were calculated and statistical significance was determined using the student's paired $\mathrm{t}$-test.

\section{Determination of NHEJ-mediated and HDR-mediated knockin by PCR and Sanger sequencing}

Human iPSCs were harvested at day 3 after cotransfection of Cas9/sgRNA and donors for DNA extraction. The CTNNB1 and PRDM14 target sequences were amplified with KAPA HiFi DNA polymerase by PCR twice. For the first-round PCR at CTNNB1 locus, CTNNB1 forward primer-F1 (GTGGCCTGGCACTG AGTAAT) and CTNNB1 reverse primer-R1 (CTCAGCAACTCTACAGGCCA) were used with the PCR cycling condition being $98{ }^{\circ} \mathrm{C}$ for $5 \mathrm{~min}$, followed by $98{ }^{\circ} \mathrm{C}$ for $5 \mathrm{~s}, 68^{\circ} \mathrm{C}$ for $1 \mathrm{~min}$ for 30 cycles. The bands in the size range of 2-4kb were cut out and purified using the GeneJET Gel Extraction Kit. For second-round PCR, $1 \mathrm{ng}$ of purified primary PCR products was amplified using the same pair of primers and cycling conditions. The bands in the range of 2-4 kb from the second PCR were purified and cloned into the pJET vector. Approximately 50 individual bacterial colonies from each condition were picked for Sanger sequencing. Clones with high-quality sequencing data at both ends were aligned with expected HDR knockin sequence and donor plasmid sequence by BLAST. Clones with wild-type gDNA insertion were reported. Similar procedure was carried 
out for the PRDM14 locus with PRDM14 forward primerF1 (CCAGCCTGCAATCTGCTTTT) and PRDM14 reverse primer-R1 (gccAACTGCAGGGACTTCTA) for the first-round PCR and PRDM14 forward primer-F2 (GACC AGGAGTGCTCTATGGC) and PRDM14 reverse primerR2 (AGGAAATAGAGAGAATCCGAATCTC) for the second-round PCR. The annealing temperature of $64{ }^{\circ} \mathrm{C}$ was used for both first-round and second-round PCR.

\section{Examination of relative incidence of NHEJ-mediated donor plasmid insertion}

To quantitate the occurrence of NHEJ-mediated insertion of large pieces from donor plasmid, we conducted qPCR. We used one possible insertion, backboneforward insertion, as a surrogate indicator of all the possible NHEJ-mediated insertion of donor plasmid. The real-time PCR reaction system $(20 \mu \mathrm{L})$ consisted of $10 \mu \mathrm{L}$ of SYBR Green qPCR Master Mix (2X), $1 \mu \mathrm{L}$ each of F2 (qPCR-BB-forward primer2, CACTCATTAGG CACCCCAGG) and R2 (qPCR-gDNA-reverse primer2, CCCACCCTACCAACCAAGTC), and 100 ng gDNA from a cell sample from day 3 . The cycling conditions were $95{ }^{\circ} \mathrm{C}$ for $3 \mathrm{~min}$, followed by $95{ }^{\circ} \mathrm{C}$ for $15 \mathrm{~s}, 64{ }^{\circ} \mathrm{C}$ for 20 s, and $72{ }^{\circ} \mathrm{C}$ for 30 s, for 35 cycles. The GAPDH gene, a housekeeping gene, was used to normalize the qPCR data. The relative NHEJ incidence was calculated by the relative value of backbone-forward insertion divided by portion of HDR in the same sample.

\section{Single-cell cloning and genotyping of edited iPSCs}

Human iPSCs were dissociated using Accutase in order to get single-cell suspension. Live iPSCs were suspended in Accutase and did the following single-cell sorting as soon as possible. Single-cell sorted using a BD FACSAria III with a $70 \mathrm{~mm}$ nozzle under sterile conditions into 96-well plates coated with Matrigel. Each well contained mTeSR medium as well as the ROCK inhibitor Y-27632 $(10 \mu \mathrm{M})$. After sorting, plates were cultured at $37{ }^{\circ} \mathrm{C}$ with $5 \% \mathrm{CO}$. Colony formation was seen seven days post sorting. The cells were refreshed with $\mathrm{mTeSR}$ medium every two to three days.

96-well cells were dissociated using EDTA and transferred into 24-well plates 14 days after FACS. Two or three days later, part of the cells were harvested for DNA extraction using a Genomic DNA Extraction Kit (Qiagen) and the remaining cells were passaged for flow. GFP and PRDM14 sequence was amplified with KAPA HiFi DNA polymerase by PCR using the following primers: PRDM14-HA300KI-F: GACCAGGAGTGCTC TATGGC, PRDM14-HA300KI-R: AGGAAATAGAGA GAATCCGAATCTC. The PCR cycling conditions were $95{ }^{\circ} \mathrm{C}$ for $4 \mathrm{~min}$ followed by $98{ }^{\circ} \mathrm{C}$ for $5 \mathrm{~s}, 64{ }^{\circ} \mathrm{C}$ for $15 \mathrm{~s}$, $72{ }^{\circ} \mathrm{C}$ for $3 \mathrm{~min}$, for 35 cycles.

\section{Confocal microscopy}

Cover glass was coated with $0.1 \%$ geltin for $30 \mathrm{~min}$ at $37{ }^{\circ} \mathrm{C}$ and then coated with Matrigel. iPSCs were seeded on cover glass and cultured for two days. Two days later, the cells with mNeonGreen fused in frame to the Cterminus of CTNNB1 were stained with Hoechst 33258 (Sigma) and Cell Mash Deep Red (Life Technology) following the protocol. iPSCs with tdTomato fused in frame to the C-terminus of PRDM14 were fixed with $4 \%$ paraformaldehyde (Sigma), followed by staining with Phalloidin 488 (Thermo Fisher Scientific) and DAPI (Sigma). Images were taken using a Zeiss Axio Observer Z1 inverted LSM 710 NLO laser scanning confocal microscope. Different lasers were used to excite each dye. Hoechst 33258 and DAPI were excited with a 405nm DPSS laser, mNeonGreen and Phalloidin 488 were activated with the 488-nm line of a $\mathrm{Kr}$-Ar multiple laser, and CellMask Red and tdTomato with a 633-nm HeNe laser. For Hoechst 33258 and DAPI, emitted light was collected at 417-679 nm using a PMT array. For $\mathrm{mNeonGreen}$ and Phalloidin, emitted light was collected with a single PMT at 493-630 nm. For CellMask Red and tdTomato, emitted light was collected with a single PMT at $638-755 \mathrm{~nm}$.

\section{Statistics}

Data were analyzed by student's paired t-test for two groups and ANOVA for more than two groups. Clone difference between different groups was analyzed by Fisher's exact test. All the values were shown as mean \pm SEM (standard error of the mean).

\section{Additional file}

Additional file 1: Supplemental Table and Figures. (PDF 5937 kb)

\section{Acknowledgements}

The authors are grateful to Monica Romero for help with the imaging in the LLUSM Advanced Imaging and Microscopy Core with support of NSF Grant MRI-DBI 0923559 and the Loma Linda University School of Medicine.

\section{Funding}

This work was supported by the Ministry of Science and Technology of China (2015CB964902, 2013CB966902, and 2012CB966601), the National Natural Science Foundation of China (81500148, 81570164, and 81421002), the Loma Linda University School of Medicine GCAT grant (2015), and Telemedicine and Advanced Technology Research Center (W81XWH-08-1-0697).

\section{Availability of data and materials}

Not applicable.

\section{Authors' contributions}

XBZ conceived of and supervised the study. JPZ, XLL, GHL, WC, CA, LZ, WW, YWF, JX, and XBZ conducted the experiments. JPZ, XLL, DB, GDB, WY, TC, and $X B Z$ analyzed the results. JPZ, XLL, TC, and XBZ wrote the paper. All authors reviewed the manuscript. All authors read and approved the final manuscript. 


\section{Competing interests}

The authors declare that they have no competing interests.

\section{Ethics approval and consent to participate}

This study did not require ethics approval.

\begin{abstract}
Author details
${ }^{1}$ State Key Laboratory of Experimental Hematology, Tianjin, China. ${ }^{2}$ Institute of Hematology and Blood Disease Hospital, Chinese Academy of Medical Sciences and Peking Union Medical College, Tianjin, China. ${ }^{3}$ Center for Stem Cell Medicine, Chinese Academy of Medical Sciences, Tianjin, China. ${ }^{4}$ Department of Stem Cell \& Regenerative Medicine, Peking Union Medical College, Tianjin, China. ${ }^{5}$ Collaborative Innovation Center for Cancer Medicine, Tianjin, China. ${ }^{6}$ Tianjin Key Laboratory of Blood Cell Therapy and Technology, Tianjin, China. ${ }^{7}$ Division of Regenerative Medicine MC1528B, Department of Medicine, Loma Linda University, 11234 Anderson Street, Loma Linda, CA 92354, USA. ${ }^{8}$ Department of Orthopaedic Surgery, Loma Linda University, Loma Linda, CA, USA.
\end{abstract}

Received: 27 October 2016 Accepted: 27 January 2017 Published online: 20 February 2017

\section{References}

1. Gaj T, Gersbach CA, Barbas 3rd CF. ZFN, TALEN, and CRISPR/Cas-based methods for genome engineering. Trends Biotechnol. 2013;31:397-405.

2. Hsu PD, Lander ES, Zhang F. Development and applications of CRISPR-Cas9 for genome engineering. Cell. 2014;157:1262-78.

3. Wang $H$, Yang H, Shivalila CS, Dawlaty MM, Cheng AW, Zhang F, et al. Onestep generation of mice carrying mutations in multiple genes by CRISPR/ Cas-mediated genome engineering. Cell. 2013;153:910-8.

4. Rouet $\mathrm{P}$, Smih F, Jasin M. Expression of a site-specific endonuclease stimulates homologous recombination in mammalian cells. Proc Natl Acad Sci U S A. 1994;91:6064-8.

5. Bollag RJ, Waldman AS, Liskay RM. Homologous recombination in mammalian cells. Annu Rev Genet. 1989;23:199-225.

6. Bibikova M, Beumer K, Trautman JK, Carroll D. Enhancing gene targeting with designed zinc finger nucleases. Science. 2003;300:764.

7. Mali $P$, et al. RNA-guided human genome engineering via Cas9. Science. 2013;339:823-6.

8. Jin Y, Park J, Jang J, Kang S. Multiplex genome engineering of Amyotrophic lateral sclerosis mutant SOD1 gene using CRISPR/Cas9 systems. FEBS J. 2015;282:178.

9. Wright AV, Nunez JK, Doudna JA. Biology and applications of CRISPR systems: harnessing nature's toolbox for genome engineering. Cell. 2016; 164:29-44.

10. Hou Z, Zhang Y, Propson NE, Howden SE, Chu LF, Sontheimer EJ, et al. Efficient genome engineering in human pluripotent stem cells using Cas9 from Neisseria meningitidis. Proc Natl Acad Sci U S A. 2013;110:15644-9.

11. Ran FA, Cong L, Yan WX, Scott DA, Gootenberg JS, Kriz AJ, et al. In vivo genome editing using Staphylococcus aureus Cas9. Nature. 2015;520:186-91.

12. Zetsche B, Gootenberg JS, Abudayyeh OO, Slaymaker IM, Makarova KS, Essletzbichler P, et al. Cpf1 is a single RNA-guided endonuclease of a class 2 CRISPR-Cas system. Cell. 2015;163:759-71.

13. Deltcheva E, Chylinski K, Sharma CM, Gonzales K, Chao Y, Pirzada ZA, et al. CRISPR RNA maturation by trans-encoded small RNA and host factor RNase III. Nature. 2011;471:602-7.

14. Jinek M, Chylinski K, Fonfara I, Hauer M, Doudna JA, Charpentier E. A programmable dual-RNA-guided DNA endonuclease in adaptive bacterial immunity. Science. 2012;337:816-21.

15. Fu Y, Sander JD, Reyon D, Cascio VM, Joung JK. Improving CRISPR-Cas nuclease specificity using truncated guide RNAs. Nat Biotechnol. 2014;32:279-84.

16. Zhang JP, Li XL, Neises A, Chen W, Hu LP, Ji GZ, et al. Different effects of sgRNA length on CRISPR-mediated gene knockout efficiency. Sci Rep. 2016;6:28566.

17. Doench JG, Hartenian E, Graham DB, Tothova Z, Hegde M, Smith I, et al. Rational design of highly active sgRNAs for CRISPR-Cas9-mediated gene inactivation. Nat Biotechnol. 2014;32:1262-7.

18. Graham DB, Root DE. Resources for the design of CRISPR gene editing experiments. Genome Biol. 2015;16:260.
19. Doench JG, Fusi N, Sullender M, Hegde M, Vaimberg EW, Donovan KF, et al. Optimized sgRNA design to maximize activity and minimize off-target effects of CRISPR-Cas9. Nat Biotechnol. 2016;34:184-91.

20. Byrne SM, Ortiz L, Mali P, Aach J, Church GM. Multi-kilobase homozygous targeted gene replacement in human induced pluripotent stem cells. Nucleic Acids Res. 2015;43, e21.

21. Zhu Z, Verma N, Gonzalez F, Shi ZD, Huangfu D. A CRISPR/Cas-mediated selection-free knockin strategy in human embryonic stem cells. Stem Cell Rep. 2015:4:1103-11.

22. Maresca M, Lin VG, Guo N, Yang Y. Obligate ligation-gated recombination (ObLiGaRe): custom-designed nuclease-mediated targeted integration through nonhomologous end joining. Genome Res. 2013;23:539-46.

23. Cristea S, Freyvert $Y$, Santiago Y, Holmes MC, Urnov FD, Gregory PD, et al. In vivo cleavage of transgene donors promotes nuclease-mediated targeted integration. Biotechnol Bioeng. 2013;110:871-80.

24. Auer TO, Duroure K, De Cian A, Concordet JP, Del Bene F. Highly efficient CRISPR/Cas9-mediated knock-in in zebrafish by homology-independent DNA repair. Genome Res. 2014;24:142-53.

25. Kimura Y, Hisano Y, Kawahara A, Higashijima S. Efficient generation of knock-in transgenic zebrafish carrying reporter/driver genes by CRISPR/Cas9mediated genome engineering. Sci Rep. 2014:4:6545.

26. He X, Tan C, Wang F, Wang Y, Zhou R, Cui D, et al. Knock-in of large reporter genes in human cells via CRISPR/Cas9-induced homologydependent and independent DNA repair. Nucleic Acids Res. 2016;44, e85.

27. Hisano Y, Sakuma T, Nakade S, Ohga R, Ota S, Okamoto H, et al. Precise inframe integration of exogenous DNA mediated by CRISPR/Cas9 system in zebrafish. Sci Rep. 2015;5:8841.

28. Nakade S, Tsubota T, Sakane Y, Kume S, Sakamoto N, Obara M, et al. Microhomology-mediated end-joining-dependent integration of donor DNA in cells and animals using TALENs and CRISPR/Cas9. Nat Commun 2014:5:5560

29. Sakuma T, Nakade S, Sakane Y, Suzuki KT, Yamamoto T. MMEJ-assisted gene knock-in using TALENs and CRISPR-Cas9 with the PITCh systems. Nat Protoc. 2016;11:118-33.

30. Yang L, Guell M, Byrne S, Yang JL, De Los AA, Mali P, et al. Optimization of scarless human stem cell genome editing. Nucleic Acids Res. 2013;41:9049-61.

31. Chen F, Pruett-Miller SM, Huang Y, Gjoka M, Duda K, Taunton J, et al. Highfrequency genome editing using ssDNA oligonucleotides with zinc-finger nucleases. Nat Methods. 2011;8:753-5.

32. Yang $H$, Wang $H$, Shivalila CS, Cheng AW, Shi L, Jaenisch R. One-step generation of mice carrying reporter and conditional alleles by CRISPR/Casmediated genome engineering. Cell. 2013;154:1370-9.

33. Maruyama T, Dougan SK, Truttmann MC, Bilate AM, Ingram JR, Ploegh HL. Increasing the efficiency of precise genome editing with CRISPR-Cas9 by inhibition of nonhomologous end joining. Nat Biotechnol. 2015;33:538-42.

34. Bottcher R, Hollmann M, Merk K, Nitschko V, Obermaier C, Philippou-Massier J, et al. Efficient chromosomal gene modification with CRISPR/cas9 and PCR-based homologous recombination donors in cultured Drosophila cells. Nucleic Acids Res. 2014:42, e89.

35. Moehle EA, Rock JM, Lee YL, Jouvenot Y, DeKelver RC, Gregory PD, et al. Targeted gene addition into a specified location in the human genome using designed zinc finger nucleases. Proc Natl Acad Sci U S A. 2007;104:3055-60.

36. Hockemeyer D, Soldner F, Beard C, Gao Q, Mitalipova M, DeKelver RC, et al. Efficient targeting of expressed and silent genes in human ESCS and iPSCS using zinc-finger nucleases. Nat Biotechnol. 2009:27:851-7.

37. Elliott B, Richardson C, Winderbaum J, Nickoloff JA, Jasin M. Gene conversion tracts from double-strand break repair in mammalian cells. Mol Cell Biol. 1998;18:93-101.

38. Orlando SJ, Santiago Y, DeKelver RC, Freyvert Y, Boydston EA, Moehle EA, et al. Zinc-finger nuclease-driven targeted integration into mammalian genomes using donors with limited chromosomal homology. Nucleic Acids Res. 2010;38, e152

39. Beumer KJ, Trautman JK, Mukherjee K, Carroll D. Donor DNA utilization during gene targeting with zinc-finger nucleases. G3 (Bethesda). 2013;3:657-64.

40. Urnov FD, Miller JC, Lee YL, Beausejour CM, Rock JM, Augustus S, et al. Highly efficient endogenous human gene correction using designed zincfinger nucleases. Nature. 2005;435:646-51.

41. Huang $X$, Wang $Y$, Yan W, Smith $C$, Ye Z, Wang J, et al. Production of genecorrected adult beta globin protein in human erythrocytes differentiated from patient iPSCs after genome editing of the sickle point mutation. Stem Cells. 2015;33:1470-9. 
42. Howden SE, Maufort JP, Duffin BM, Elefanty AG, Stanley EG, Thomson JA. Simultaneous reprogramming and gene correction of patient fibroblasts. Stem cell Rep. 2015;5:1109-18.

43. Su RJ, Baylink DJ, Neises A, Kiroyan JB, Meng X, Payne KJ, et al. Efficient generation of integration-free ips cells from human adult peripheral blood using BCL-XL together with Yamanaka factors. PLoS One. 2013;8, e64496.

44. Shaner NC, Lambert GG, Chammas A, Ni Y, Cranfill PJ, Baird MA, et al. A bright monomeric green fluorescent protein derived from Branchiostoma lanceolatum. Nat Methods. 2013;10:407-9.

45. Nakaki F, Saitou M. PRDM14: a unique regulator for pluripotency and epigenetic reprogramming. Trends Biochem Sci. 2014;39:289-98.

46. Robert F, Barbeau M, Ethier S, Dostie J, Pelletier J. Pharmacological inhibition of DNA-PK stimulates Cas9-mediated genome editing. Genome Med. 2015;7:93.

47. Yu C, Liu Y, Ma T, Liu K, Xu S, Zhang Y, et al. Small molecules enhance CRISPR genome editing in pluripotent stem cells. Cell Stem Cell. 2015;16:142-7.

48. Lin S, Staahl BT, Alla RK, Doudna JA. Enhanced homology-directed human genome engineering by controlled timing of CRISPR/Cas9 delivery. Elife. 2014;3, e04766.

49. Pinder J, Salsman J, Dellaire G. Nuclear domain 'knock-in' screen for the evaluation and identification of small molecule enhancers of CRISPR-based genome editing. Nucleic Acids Res. 2015;43:9379-92.

50. Song J, Yang D, Xu J, Zhu T, Chen YE, Zhang J. RS-1 enhances CRISPR/Cas9and TALEN-mediated knock-in efficiency. Nat Commun. 2016;7:10548.

51. Chu VT, Weber T, Wefers B, Wurst W, Sander S, Rajewsky K, et al. Increasing the efficiency of homology-directed repair for CRISPR-Cas9-induced precise gene editing in mammalian cells. Nat Biotechnol. 2015;33:543-8.

52. Baldin V, Lukas J, Marcote MJ, Pagano M, Draetta G. Cyclin D1 is a nuclear protein required for cell cycle progression in G1. Genes Dev. 1993;7:812-21.

53. Richardson CD, Ray GJ, DeWitt MA, Curie GL, Corn JE. Enhancing homologydirected genome editing by catalytically active and inactive CRISPR-Cas9 using asymmetric donor DNA. Nat Biotechnol. 2016;34:339-44.

54. Zheng Q, Cai X, Tan MH, Schaffert S, Arnold CP, Gong X, et al. Precise gene deletion and replacement using the CRISPR/Cas9 system in human cells. Biotechniques. 2014;57:115-24.

55. Sugawara N, Ira G, Haber JE. DNA length dependence of the single-strand annealing pathway and the role of Saccharomyces cerevisiae RAD59 in double-strand break repair. Mol Cell Biol. 2000;20:5300-9.

56. Jayathilaka K, Sheridan SD, Bold TD, Bochenska K, Logan HL, Weichselbaum RR, et al. A chemical compound that stimulates the human homologous recombination protein RAD51. Proc Natl Acad Sci U S A. 2008;105:15848-53.

57. Heyer WD, Ehmsen KT, Liu J. Regulation of homologous recombination in eukaryotes. Annu Rev Genet. 2010;44:113-39.

58. Rothkamm K, Kruger I, Thompson LH, Lobrich M. Pathways of DNA double-strand break repair during the mammalian cell cycle. Mol Cell Biol. 2003;23:5706-15.

59. Gutschner T, Haemmerle M, Genovese G, Draetta GF, Chin L. Posttranslational regulation of Cas9 during G1 enhances homology-directed repair. Cell Rep. 2016;14:1555-66.

60. Howden SE, McColl B, Glaser A, Vadolas J, Petrou S, Little MH, et al. A Cas9 variant for efficient generation of indel-free knockin or gene-corrected human pluripotent stem cells. Stem cell Rep. 2016;7:508-17.

61. Zwaka TP, Thomson JA. Homologous recombination in human embryonic stem cells. Nat Biotechnol. 2003;21:319-21.

62. Zou J, Mali P, Huang X, Dowey SN, Cheng L. Site-specific gene correction of a point mutation in human IPS cells derived from an adult patient with sickle cell disease. Blood. 2011;118:4599-608.

63. Arbab M, Srinivasan S, Hashimoto T, Geijsen N, Cloning-free SRI, CRISPR. Stem Cell Rep. 2015;5:908-17.

64. Kim S, Kim D, Cho SW, Kim J, Kim JS. Highly efficient RNA-guided genome editing in human cells via delivery of purified Cas9 ribonucleoproteins. Genome Res. 2014;24:1012-9.

65. Schumann K, Lin S, Boyer E, Simeonov DR, Subramaniam M, Gate RE, et al. Generation of knock-in primary human T cells using Cas9 ribonucleoproteins. Proc Natl Acad Sci U S A. 2015;112:10437-42.

66. Hendel A, Bak RO, Clark JT, Kennedy AB, Ryan DE, Roy S, et al. Chemically modified guide RNAs enhance CRISPR-Cas genome editing in human primary cells. Nat Biotechnol. 2015;33:985-9.

67. Montague TG, Cruz JM, Gagnon JA, Church GM, Valen E. CHOPCHOP: a CRISPR/Cas9 and TALEN web tool for genome editing. Nucleic Acids Res. 2014;42:W401-407.

\section{Submit your next manuscript to BioMed Central and we will help you at every step:}

- We accept pre-submission inquiries

- Our selector tool helps you to find the most relevant journal

- We provide round the clock customer support

- Convenient online submission

- Thorough peer review

- Inclusion in PubMed and all major indexing services

- Maximum visibility for your research

Submit your manuscript at www.biomedcentral.com/submit 